OPEN ACCESS

Edited by:

Fahmi Khalifa,

Mansoura University, Egypt

Reviewed by:

Alexandra L. Young,

University College London,

United Kingdom

Yaser A. EINakieb,

University of Louisville, United States

*Correspondence:

Yalin Wang

ylwang@asu.edu

tThese authors have contributed equally to this work

Specialty section:

This article was submitted to

Brain Imaging Methods,

a section of the journal

Frontiers in Neuroscience

Received: 19 February 2021

Accepted: 15 July 2021

Published: 06 August 2021

Citation:

Wu J, Dong Q, Gui J, Zhang J,

Su Y, Chen K, Thompson PM,

Caselli RJ, Reiman EM, Ye J and

Wang Y (2021) Predicting Brain

Amyloid Using Multivariate

Morphometry Statistics, Sparse Coding, and Correntropy: Validation in 1,101 Individuals From the ADNI

and OASIS Databases.

Front. Neurosci. 15:669595. doi: 10.3389/fnins.2021.669595

\section{Predicting Brain Amyloid Using Multivariate Morphometry Statistics, Sparse Coding, and Correntropy: Validation in 1,101 Individuals From the ADNI and OASIS Databases}

\author{
Jianfeng Wu't, Qunxi Dong ${ }^{1,2 t}$, Jie Gui ${ }^{3}$, Jie Zhang', Yi Su4 ${ }^{4}$ Kewei Chen ${ }^{4}$, \\ Paul M. Thompson ${ }^{5}$, Richard J. Caselli ${ }^{6}$, Eric M. Reiman ${ }^{4}$, Jieping $\mathrm{Ye}^{7}$ and \\ Yalin Wang ${ }^{1 *}$ for the Alzheimer's Disease Neuroimaging Initiative
}

${ }^{1}$ School of Computing, Informatics, and Decision Systems Engineering, Arizona State University, Tempe, AZ, United States, 2 Institute of Engineering Medicine, Beijing Institute of Technology, Beijing, China, ${ }^{3}$ School of Cyber Science and Engineering, Southeast University, Nanjing, China, ${ }^{4}$ Banner Alzheimer's Institute, Phoenix, AZ, United States, ${ }^{5}$ Imaging Genetics Center, Stevens Neuroimaging and Informatics Institute, University of Southern California, Marina del Rey, CA, United States, ${ }^{6}$ Department of Neurology, Mayo Clinic Arizona, Scottsdale, AZ, United States, ${ }^{7}$ Department of Computational Medicine and Bioinformatics, University of Michigan, Ann Arbor, MI, United States

Biomarker assisted preclinical/early detection and intervention in Alzheimer's disease (AD) may be the key to therapeutic breakthroughs. One of the presymptomatic hallmarks of $A D$ is the accumulation of beta-amyloid $(A \beta)$ plaques in the human brain. However, current methods to detect $A \beta$ pathology are either invasive (lumbar puncture) or quite costly and not widely available (amyloid PET). Our prior studies show that magnetic resonance imaging (MRI)-based hippocampal multivariate morphometry statistics (MMS) are an effective neurodegenerative biomarker for preclinical AD. Here we attempt to use MRI-MMS to make inferences regarding brain $A \beta$ burden at the individual subject level. As MMS data has a larger dimension than the sample size, we propose a sparse coding algorithm, Patch Analysis-based Surface Correntropyinduced Sparse-coding and Max-Pooling (PASCS-MP), to generate a low-dimensional representation of hippocampal morphometry for each individual subject. Then we apply these individual representations and a binary random forest classifier to predict brain $A \beta$ positivity for each person. We test our method in two independent cohorts, 841 subjects from the Alzheimer's Disease Neuroimaging Initiative (ADNI) and 260 subjects from the Open Access Series of Imaging Studies (OASIS). Experimental results suggest that our proposed PASCS-MP method and MMS can discriminate A $\beta$ positivity in people with mild cognitive impairment $(\mathrm{MCl})[$ Accuracy $(\mathrm{ACC})=0.89(\mathrm{ADNI})]$ and in cognitively unimpaired $(\mathrm{CU})$ individuals $[\mathrm{ACC}=0.79(\mathrm{ADNI})$ and $\mathrm{ACC}=0.81(\mathrm{OASIS})]$. These results compare favorably relative to measures derived from traditional algorithms, including hippocampal volume and surface area, shape measures based on spherical harmonics (SPHARM) and our prior Patch Analysis-based Surface Sparse-coding and Max-Pooling (PASS-MP) methods.

Keywords: Alzheimer's disease, hippocampal multivariate morphometry statistics, Dictionary and Correntropyinduced Sparse Coding, beta-amyloid burden, ADNI and OASIS database 


\section{INTRODUCTION}

Alzheimer's disease (AD) is a major public health concern with the number of affected individuals expected to triple, reaching 13.8 million by the year 2050 in the United States alone (Brookmeyer et al., 2007). Current therapeutic failures in patients with dementia due to $\mathrm{AD}$ may be due to interventions that are too late, or targets that are secondary effects and less relevant to disease initiation and early progression (Hyman, 2011). Preclinical AD is now viewed as a gradual process that begins many years before the onset of clinical symptoms. Measuring brain biomarkers and intervening at preclinical $\mathrm{AD}$ stages are believed to improve the probability of therapeutic success (Brookmeyer et al., 2007; Sperling et al., 2011a; Jack et al., 2016). In the $\mathrm{A} / \mathrm{T} / \mathrm{N}$ system - a recently proposed research framework for understanding the biology of $\mathrm{AD}$ - the presence of abnormal levels of beta-amyloid $(A \beta)$ in the brain or cerebrospinal fluid (CSF) is used to define the presence of biological $\mathrm{AD}$ (Jack et al., 2016). An imbalance between production and clearance of $A \beta$ occurs early in $\mathrm{AD}$ and is typically followed by the accumulation of tau protein tangles (another key pathological hallmark of $\mathrm{AD}$ ) and neurodegeneration detectable on brain magnetic resonance imaging (MRI) scans (Hardy and Selkoe, 2002; Sperling et al., 2011a; Jack et al., 2016). Brain A $\beta$ pathology can be measured using positron emission tomography (PET) with $A \beta$-sensitive radiotracers, or in CSF. Meanwhile, ongoing research has been focused on the harmonization over different tracers (Klunk et al., 2015), and the standardization of data acquisition and preprocessing (Su et al., 2015, 2018) for amyloid PET before they can be eventually implemented for clinical use. Even so, these invasive and expensive measurements are less attractive to subjects in preclinical stage and PET scanning is also not as widely available as MRI.

Blood-based biomarkers (BBBs) are somewhat effective for inferring $A \beta$ burden in the brain and CSF, and are less expensive than imaging (Bateman et al., 2019; Janelidze et al., 2020; Palmqvist et al., 2020). Even so, structural MRI biomarkers are largely accessible, cost-effective, and widely used in $\mathrm{AD}$ imaging research as well as for clinical diagnosis. Consequently, there is great research interest in using MRI biomarkers to predict brain A $\beta$ burden (Sperling et al., 2011a; Tosun et al., 2014, 2016; Pekkala et al., 2020). Tosun et al. (2014) combine MRIbased measures of cortical shape and cerebral blood flow to predict $A \beta$ status for early-MCI individuals and achieve an $83 \%$ accuracy with the LASSO approach (least absolute shrinkage and selection operator). Pekkala et al. (2020) use brain MRI measures (volumes of the cortical gray matter, hippocampus, accumbens, thalamus, and putamen) to infer $\mathrm{A} \beta$ positivity in cognitively unimpaired (CU) subjects; they achieve a 0.70 area under the receiver operator curve (AUC) with their Disease State Index (DSI) algorithm. Although brain structural volumes are perhaps the most commonly used neuroimaging measures in AD research (Crivello et al., 2010; Reiter et al., 2017; Cacciaglia et al., 2018), surface-based subregional structure measures can offer advantages over volume measures as they contain more detailed and patient-specific shape information (Styner et al., 2004; Thompson et al., 2004; Morra et al., 2009; Qiu et al., 2009;
Shen et al., 2009; Apostolova et al., 2010; Costafreda et al., 2011; Younes et al., 2014; Dong et al., 2019, 2020b; Ching et al., 2020).

Our prior studies (Wang et al., 2010, 2011; Shi et al., 2014) propose novel multivariate morphometry statistics (MMS) and apply them to analyze APOE4 dose effects on brain structures of non-demented and CU groups from the ADNI cohort (Shi et al., 2014; Li et al., 2016; Dong et al., 2019). Our proposed MMS approach uses multivariate tensor-based morphometry (mTBM) to encode morphometry along the surface tangent direction and radial distance $(\mathrm{RD})$ to encode morphometry along the surface normal direction. This approach performs better for detecting clinically relevant group differences, relative to other TBM-based methods including those using the Jacobian determinant, the largest and smallest eigenvalues of the surface metric and the pair of eigenvalues of the Jacobian matrix (Wang et al., 2010, 2011). Our recent studies (Dong et al., 2019, 2020b) show that MMS outperforms volume measures for detecting hippocampal and ventricular deformations in groups at high risk for $\mathrm{AD}$ at the preclinical stage. Our other related work (Wu et al., 2018) has studied hippocampal morphometry in cohorts consisting of $A \beta$ positive $A D$ patients $(A \beta+A D)$ and $A \beta$ negative $C U$ subjects $(A \beta-C U)$ using the MMS measure. We find significant $A \beta+$ $A D$ vs. $A \beta-C U$ group differences, using Hotelling's $T^{2}$ tests. As MMS have a high dimension, it is not suitable for classification research directly. Therefore, we apply a Patch Analysis-based Surface Sparse-coding and Max-Pooling (PASS-MP) system for a low-dimensional representation of hippocampal MMS, and the binary group random forest classification of $A \beta+A D$ and $A \beta-$ $\mathrm{CU}$, achieving an accuracy rate of $90.48 \%$. These studies show that MMS can distinguish clinical groups with different $A \beta$ status. We have also successfully applied PASS-MP for MMS-based AD cognitive scores and autism spectrum disorder predictions (Dong et al., 2020a; Fu et al., 2021).

In this work, we optimize the objective function of the PASSMP system by introducing correntropy measure (Gui et al., 2017) and propose an improved sparse coding, dubbed as the Patch Analysis-based Surface Correntropy-induced Sparsecoding and Max-Pooling (PASCS-MP) method. PASCS-MP does not only take the advantage of the computational efficiency of PASS-MP in its new optimization strategy, but also effectively reduces the negative influence of non-Gaussian noise in the data, which tremendously improves the prediction accuracy. PASCS-MP is an unsupervised learning method to generate a low-dimensional representation for each sample. We leverage the novel PASCS-MP method on MMS to further explore hippocampal morphometry differences for the following contrasts at the individual subject level: (1) $A \beta$ positive individuals with mild cognitive impairment $(\mathrm{A} \beta+\mathrm{MCI})$ vs. $\mathrm{A} \beta$ negative individuals with mild cognitive impairment $(\mathrm{A} \beta-$ MCI) from $A D N I$, and (2) $A \beta$ positive $C U$ subjects $(A \beta+C U$ from $A D N I$ and OASIS) vs. $A \beta$ negative $C U$ subjects $(A \beta-$ CU from ADNI and OASIS). We apply the proposed PASCSMP and a binary random forest classifier to classify individuals with different $A \beta$ status. We hypothesize that our MMS-based PASCS-MP may provide stronger statistical power relative to traditional hippocampal volume, surface area and spherical harmonics (SPHARM) based hippocampal shape measurements, 
in predicting subjects' $A \beta$ status. We expect that the knowledge gained from this type of research will enrich our understanding of the relationship between hippocampal atrophy and $\mathrm{AD}$ pathology, and thus help in assessing disease burden, progression, and treatment effects.

\section{SUBJECTS AND METHODS}

\section{Subjects}

Data used in the preparation of this article were obtained from the Alzheimer's Disease Neuroimaging Initiative (ADNI) database ${ }^{1}$ and the Open Access Series of Imaging Studies (OASIS) database (Marcus et al., 2010). The ADNI was launched in 2003 as a public-private partnership, led by Principal Investigator Michael W. Weiner, MD. The primary goal of ADNI has been to test whether serial MRI, PET, other biological markers, and clinical and neuropsychological assessment can be combined to measure the progression of mild cognitive impairment (MCI) and early AD. For up-to-date information, see www.adni-info.org.

Table 1 shows demographic information we analyze from the ADNI and OASIS cohorts. From the ADNI cohort, we analyze 841 age and sex-matched subjects with florbetapir PET data and T1-weighted MR images, including $151 \mathrm{AD}$ patients, $342 \mathrm{MCI}$, and 348 asymptomatic CU individuals. Among them, all the 151 AD patients, 171 people with MCI, and $116 \mathrm{CU}$ individuals were $\mathrm{A} \beta$ positive. The remaining $171 \mathrm{MCI}$ and $232 \mathrm{CU}$ individuals were $\mathrm{A} \beta$ negative. From OASIS database, we analyze age-and-sexmatched 260 subjects with florbetapir PET data and T1-weighted MR images, including $52 \mathrm{~A} \beta$ positive $\mathrm{CU}$ and $208 \mathrm{~A} \beta$ negative $\mathrm{CU}$. To match the age and sex to the control group, we randomly select subjects from the majority group millions of times. For each selected group and control group, we analyze the age with $t$-test and sex with Chi-square test. We first select the groups, of which the $p$-value of Chi-squared test is 1 . Among these groups, we select the one with the largest $p$-value of age. In this way, we try to minimize the statistical difference in age and sex. The $p$-values of Chi-squared test for sex and the $p$-values of $t$-test for age are shown in the columns after sex and age in Table 1.

In addition to each MRI scan, we also analyze centiloid measures (Navitsky et al., 2018) from florbetapir PET data in ADNI and OASIS. Operationally, the positivity of $\mathrm{A} \beta$ biomarkers

${ }^{1}$ adni.loni.usc.edu is defined using standard cut-offs, with some efforts to reconcile differences among different $\mathrm{A} \beta$ radiotracers using a norming approach called the centiloid scale (Klunk et al., 2015; Rowe et al., 2017). ADNI florbetapir PET data are processed using AVID pipeline (Navitsky et al., 2018), and OASIS florbetapir PET data are processed using PUP (Lee et al., 2013; Su et al., 2015). Both are converted to the centiloid scales according to their respective conversion equations (Navitsky et al., 2018; Su et al., 2019). A centiloid cutoff of 37.1 is used to determine $A \beta$ positivity, this threshold corresponds to pathologically determined moderate to frequent plaques (Fleisher et al., 2011).

\section{Proposed Pipeline}

This work develops the PASCS-MP framework to predict individual $A \beta$ burden (see Figure 1 for the processing pipeline). In panel (1), hippocampal structures are segmented from registered brain MR images with FMRIB's Integrated Registration and Segmentation Tool (FIRST) from the FMRIB Software Library (FSL) (Patenaude et al., 2011; Paquette et al., 2017). Hippocampal surface meshes are constructed with the marching cubes algorithm (Lorensen and Cline, 1987). In panel (2), hippocampal surfaces are parameterized with the holomorphic flow segmentation method (Wang et al., 2007). After the surface fluid registration algorithm, the hippocampal MMS features are calculated at each surface point. We propose a PASCS-MP and classification system to refine and classify MMS patches in individuals with different $A \beta$ status. We randomly select patches on each hippocampal surface and generate a sparse code for each patch with our novel PASCS. Next, we adopt a Max-pooling operation on the learned sparse codes of these patches to generate a new representation (a vector) for each subject. Finally, we train binary random forest classifiers on individual sparse codes in people with different $A \beta$ status; we validate them with 10 -fold cross-validation. The whole system is publicly available. ${ }^{2}$

\section{Image Processing}

Firstly, we use FIRST (Patenaude et al., 2011) to segment the original MRI data and map the hippocampus substructure. After obtaining a binary segmentation of the hippocampus, we use a topology-preserving level set method (Han et al., 2003) to build surface models. Based on that, the marching cubes algorithm (Lorensen and Cline, 1987) is applied to construct

\footnotetext{
${ }^{2}$ http://gsl.lab.asu.edu/software/pass-mp/
}

TABLE 1 | Demographic information for the subjects we study from the ADNI and OASIS cohorts.

\begin{tabular}{|c|c|c|c|c|c|c|c|}
\hline Database & Group & Sex (M/F) & $p$-Value & Age & $p$-Value & MMSE & Centiloid \\
\hline \multirow[t]{5}{*}{$\operatorname{ADNI}(n=841)$} & $A \beta+A D(n=151)$ & $79 / 72$ & & $74.6 \pm 7.8$ & & $22.6 \pm 3.1$ & $86.3 \pm 27.4$ \\
\hline & $A \beta+M C l(n=171)$ & $92 / 79$ & 1.00 & $74.1 \pm 7.4$ & 0.90 & $27.7 \pm 1.7$ & $76.8 \pm 26.4$ \\
\hline & $A \beta-M C l(n=171)$ & $92 / 79$ & & $74.0 \pm 7.4$ & & $28.3 \pm 1.6$ & $8.9 \pm 14.9$ \\
\hline & $A \beta+C U(n=116)$ & $45 / 71$ & 1.00 & $75.9 \pm 6.1$ & 0.78 & $28.9 \pm 1.1$ & $71.1 \pm 26.4$ \\
\hline & $A \beta-C U(n=232)$ & $90 / 142$ & & $75.7 \pm 6.3$ & & $29.0 \pm 1.3$ & $7.5 \pm 14.5$ \\
\hline \multirow[t]{2}{*}{ OASIS $(n=260)$} & $A \beta+C U(n=52)$ & $22 / 30$ & 1.00 & $70.5 \pm 7.5$ & 0.08 & $29.0 \pm 1.3$ & $71.4 \pm 20.9$ \\
\hline & $A \beta-C U(n=208)$ & $88 / 120$ & & $68.5 \pm 6.8$ & & $29.0 \pm 1.3$ & $8.5 \pm 9.5$ \\
\hline
\end{tabular}

Values are mean $\pm S D$ where applicable. 


\section{(1) Hippocampal structure extraction from MR images with FIRST}

A

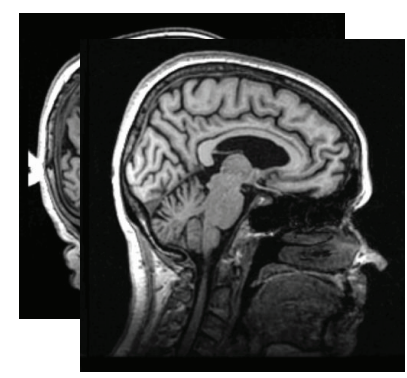

MRI scans
B

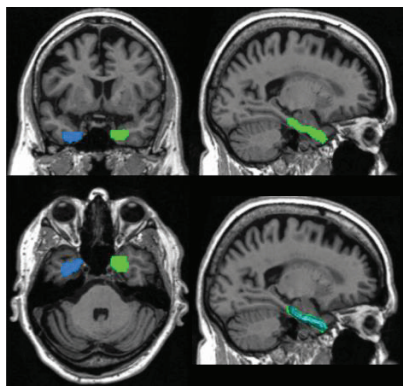

Hippocampal Segmentation
C

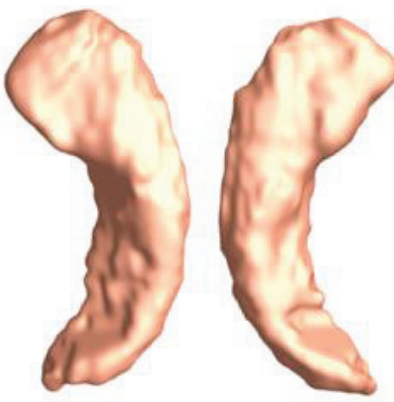

Smoothed Surface

(2) Multivariate Morphometry Statistics and our proposed machine learning system involving sparse coding and random forest methods to classify $A B+$ and $A B$-samples

A

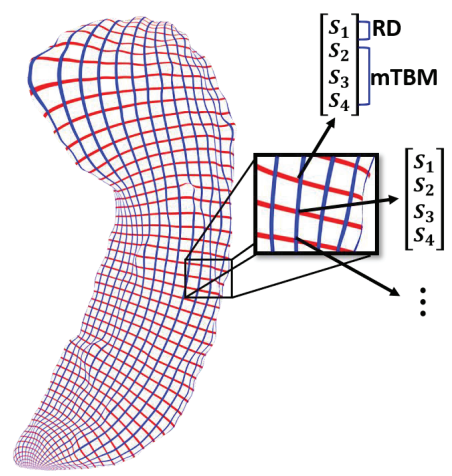

Multivariate Morphometry Statistics

D

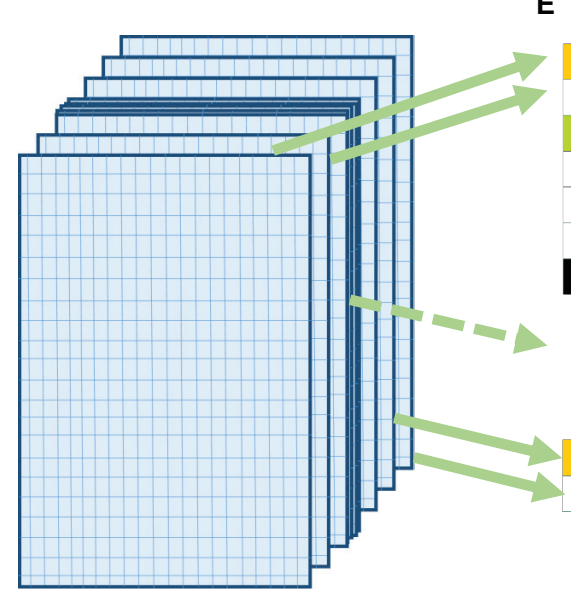

Sparse Patch-based Features
B

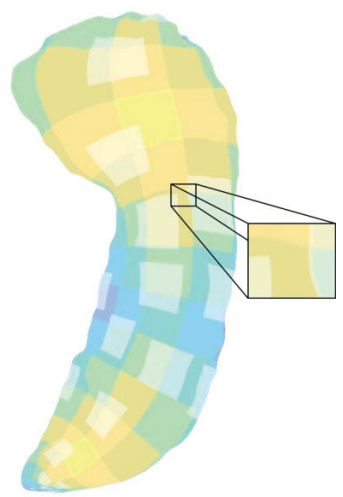

Patch Selection
C

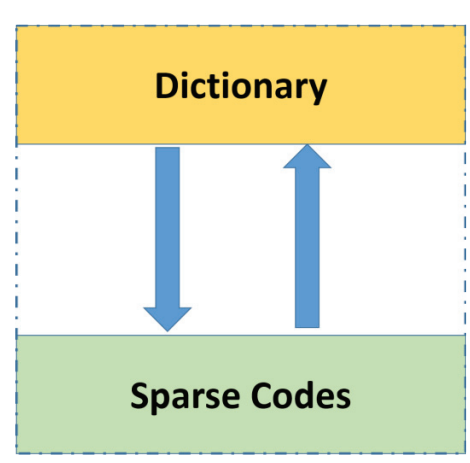

Correntropy-induced Stochastic Coordinate Coding
E

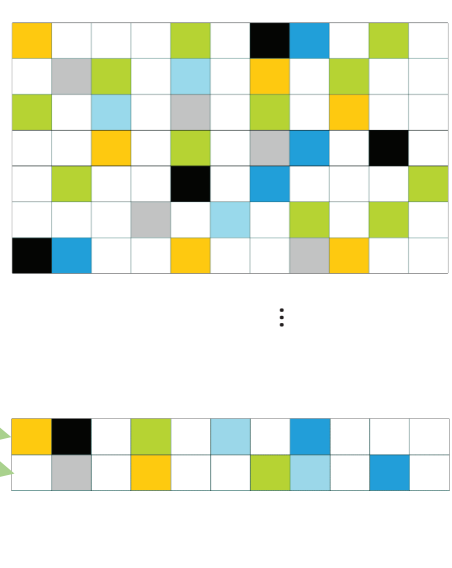

Max-Pooling
F

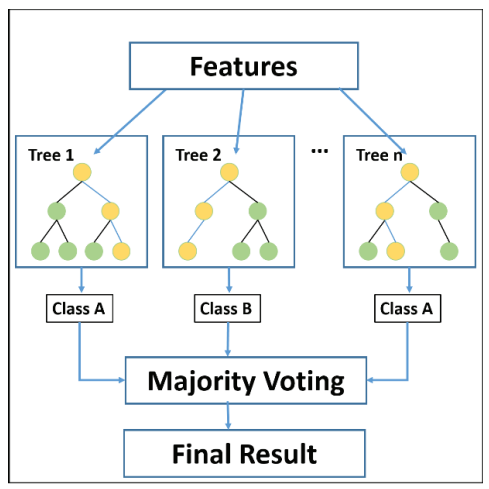

Classification: Random Forest

FIGURE 1 | Panel (1) shows hippocampal surfaces generated from brain MRI scans. In subfigure (A) of panel (2), surface-based multivariate morphometry statistics (MMS) are calculated after fluid registration of surface coordinates across subjects. MMS is a $4 \times 1$ vector on each vertex, including radial distance (scalar) and multivariate tensor-based morphometry $(3 \times 1$ vector). In subfigures $\mathbf{( B , C )}$, we randomly select patches on each hippocampal surface and generate a sparse code for each patch with our novel Patch Analysis-based Surface Correntropy-induced Sparse-coding (PASCS) method. In subfigures (D,E), we apply the max pooling operation to the learned sparse codes to generate a new representation (a vector) for each subject. In subfigure (F), we train binary random forest classifiers on these representations and validate them with 10 -fold cross-validation. 
triangular surface meshes. Then, to reduce the noise from MR image scanning and to overcome partial volume effects, surface smoothing is applied consistently to all surfaces. Our surface smoothing process consists of mesh simplification using progressive meshes (Hoppe, 1996) and mesh refinement by the Loop subdivision surface method (Loop, 1987). Similar procedures adopted in a number of our prior studies (Wang et al., 2010, 2012; Colom et al., 2013; Luders et al., 2013; Monje et al., 2013; Shi et al., 2013a,b, 2015) have shown that the smoothed meshes are accurate approximations to the original surfaces, with a higher signal-to-noise ratio (SNR).

To facilitate hippocampal shape analysis, we generate a conformal grid $(150 \times 100)$ on each surface, which is used as a canonical space for surface registration. On each hippocampal surface, we compute its conformal grid with a holomorphic 1 -form basis (Wang et al., 2007, 2010). We adopt surface conformal representation (Shi et al., 2013a, 2015) to obtain surface geometric features for automatic surface registration. This consists of the conformal factor and mean curvature, encoding both intrinsic surface structure and information on its three-dimensional embedding. After we compute these two local features at each surface point, we compute their summation and then linearly scale the dynamic range of the summation into the range $0-255$, to obtain a feature image for the surface. We further register each hippocampal surface to a common template surface. With surface conformal parameterization and conformal representation, we generalize the well-studied image fluid registration algorithm (Bro-Nielsen and Gramkow, 1996; D'Agostino et al., 2003) to general surfaces. Furthermore, most of the image registration algorithms in the literature are not symmetric, i.e., the correspondences between the two images depending on which image is assigned as the deforming image and which is the non-deforming target image. An asymmetric algorithm can be problematic as it tends to penalize the expansion of image regions more than shrinkage (Rey et al., 2002). Thus, in our system, we further extend the surface fluid registration method to an inverse-consistent framework (Leow et al., 2005). The obtained surface registration is diffeomorphic. For details of our inverse-consistent surface fluid registration method, we refer to (Shi et al., 2013a).

\section{Surface-Based Morphometry Feature Extraction}

After parameterization and registration, we establish a one-toone correspondence map between hippocampal surfaces. This makes it effective for us to compare and analyze surface data. Besides, each surface has the same number of vertices $(150 \times$ 100) as shown in panel (2) of Figure 1. The intersection of the red curve and the blue curve is a surface vertex, and at each vertex, we adopt two features, the $\mathrm{RD}$ and the surface metric tensor used in mTBM. The RD (a scalar at each vertex) represents the thickness of the shape at each vertex to the medical axis (Pizer et al., 1999; Thompson et al., 2004), this reflects the surface differences along the surface normal directions. The medial axis is determined by the geometric center of the isoparametric curve on the computed conformal grid (Wang et al., 2011). The axis is perpendicular to the isoparametric curve, so the thickness can be easily calculated as the Euclidean distance between the core and the vertex on the curve. The mTBM statistics (a $3 \times 1$ vector at each vertex) have been frequently studied in our prior work (Shi et al., 2013b, 2015; Wang et al., 2009, 2010). They measure local surface deformation along the surface tangent plane and show improved signal detection sensitivity relative to more standard tensor-based morphometry (TBM) measures computed as the determinant of the Jacobian matrix (Wang et al., 2013). RD and mTBM jointly form a new feature, known as the surface MMS. Therefore, MMS is a $4 \times 1$ vector at each vertex. The surface of the hippocampus in each brain hemisphere has 15, 000 vertices, so the feature dimensionality for each hippocampus in each subject is 60,000 .

\section{Surface Feature Dimensionality Reduction}

The above mentioned vertex-wise surface morphometry feature, MMS, is a high-fidelity measure to describe the local deformation of the surface and can provide detailed localization and visualization of regional atrophy or expansion (Yao et al., 2018) and development (Thompson et al., 2000). However, the high dimensionality of such features is likely to cause problems for classification. Feature reduction methods proposed by Davatzikos et al. (2008) and Sun et al. (2009) may ignore the intrinsic properties of a structure's regional morphometry. Therefore, we introduce the following feature reduction method for the vertex-wise surface morphometry features.

The surface MMS feature dimension is typically much larger than the number of subjects, i.e., the so-called high dimensionsmall sample problem. To extract useful surface features and reduce the dimension before making predictions, this work first randomly generates square windows on each surface to obtain a collection of small image patches with different amounts of overlap. In our prior AD studies (Zhang et al., 2016a,b; Wu et al., 2018), we discuss the most suitable patch size and number. Therefore, in this work, we adopt the same optimal experimental settings, as 1,008 patches (patch size $=10 \times 10$ vertices) for each subject (504 patches for each side of the hippocampal surface). As these patches are allowed to overlap, a vertex may be contained in several patches. The zoomedin window in subfigure B of panel (2) in Figure 1 [panel (2), B] shows overlapping areas on selected patches. After that, we use the technique of sparse coding and dictionary learning (Mairal et al., 2009) to learn meaningful features. Dictionary learning has been successful in many image processing tasks as it can concisely model natural image patches. In this work, we propose a novel sparse coding and dictionary learning method with an $l_{1}$-regularized correntropy loss function named Correntropy-induced Sparse-coding (CS), which is expected to improve the computational efficiency compared to Stochastic Coordinate Coding (SCC) (Lin et al., 2014). Formally speaking, correntropy is a generalized similarity measure between two scalar random variables $\mathrm{U}$ and $\mathrm{V}$, which is defined by $\mathcal{V}_{\sigma}(U, V)=\mathbb{E} \mathcal{K}_{\sigma}(U, V)$. Here, $\mathcal{K}_{\sigma}$ is a Gaussian kernel given by $\mathcal{K}_{\sigma}(U, V)=\exp \left\{-(\mathrm{u}-\mathrm{v})^{2} / \sigma^{2}\right\}$ with the scale parameter $\sigma>0$, (u-v) being a realization of (U, V) (Feng et al., 2015; Gui et al., 2017). Utilizing the correntropy measure as a loss 
function will reduce the negative influence of non-Gaussian noise in the data.

Classical dictionary learning techniques (Olshausen and Field, 1997; Lee et al., 2007) consider a finite training set of feature maps, $X=\left(x_{1}, x_{2}, x_{n}\right)$ in $R^{p \times n}$. In our study, $X$ is the set of MMS features from $n$ surface patches of all the samples. All the MMS features on each surface patch, $x_{i}$, is reshaped to a $p$ dimensional vector. And we desire to generate a new set of sparse codes, $Z=\left(z_{1}, z_{2}, z_{n}\right)$ in $R^{m \times n}$ for these features. Therefore, we aim to optimize the empirical cost function as Eq. 1 .

$$
f\left(D, z_{i}\right) \triangleq \sum_{i=1}^{n} l\left(x_{i}, D, z_{i}\right)
$$

where $D \in R^{p \times m}$ is the dictionary and $z_{i} \in R^{m}$ is the sparse code of each feature vector. $l\left(x_{i}, D, z_{i}\right)$ is the loss function that measures how well the dictionary $D$ and the sparse code $z_{i}$ can represent the feature vector $x_{i}$. Then, $x_{i}$ can be approximated by $x_{i}=D z_{i}$. In this way, we convert the $p$-dimensional feature vector, $x_{i}$, to a $m$-dimensional sparse code, $z_{i}$, where $m$ is the dimensionality of the sparse code and the dimensionality could be arbitrary. In this work, we introduce the correntropy measure (Gui et al., 2017) to the loss function and define the $l_{1}$-sparse coding optimization problem as Eq. 2

$$
\min _{D, z_{i}} \frac{1}{2} \sum_{i=1}^{n} \exp \left(-\frac{\left\|D z_{i}-x_{i}\right\|_{2}^{2}}{\sigma^{2}}\right)+\lambda \sum_{i=1}^{n}\left\|z_{i}\right\|_{1}
$$

where $\lambda$ is the regularization parameter, $\sigma$ is the kernel size that controls all properties of correntropy. $\|\cdot\|_{2}$ and $\|\cdot\|_{1}$ are the $l_{2}$-norm and $l_{1}$-norm and $\exp ()$ represents the exponential function. The first part of the loss function measures the degree of the image patches' goodness and the correntropy may help remove outliers. Meanwhile, the second part is well known as the $l_{1}$ penalty $(\mathrm{Fu}, 1998)$ that can yield a sparse solution for $z_{i}$ and select robust and informative features. Specifically, there are $m$ columns (atoms) in the dictionary $D$ and each atom is $d_{j} \in R^{p}, j=1,2, \ldots, m$. To avoid $D$ from being arbitrarily large and leading to arbitrary scaling of the sparse codes, we constrain each $l_{2}$-norm of each atom in the dictionary no larger than one. We will let $C$ become the convex set of matrices verifying the constraint as Eq. 3.

$$
\mathrm{C} \triangleq\left\{D \in R^{p \times m} s . t . \forall j=1,2,, m, d_{j}^{T} d_{j} \leq 1\right\}
$$

Note that, the empirical problem cost $f\left(D, z_{i}\right)$ is not convex when we jointly consider the dictionary $D$ and the coefficients $Z$. But the function is convex concerning each of the two variables, $D$, and $Z$, when the other one is fixed. Since it takes much time to solve $D$ and $Z$ when dealing with large-scale data sets and a large-size dictionary, we adopt the framework in the stochastic coordinate coding (SCC) algorithm (Lin et al., 2014), which can dramatically reduce the computational cost of the sparse coding, while keeping a comparable performance.

To solve this optimization problem, we reformulate the first part of the equation by the half-quadratic technique
(Nikolova and $\mathrm{Ng}, 2006$ ) and then the objective can be solved as the minimization problem Eq. 4:

$$
\begin{aligned}
& \underset{D, z_{i}}{\min } \frac{1}{2} \sum_{i=1}^{n} h_{i}\left\|D z_{i}-x_{i}\right\|_{2}^{2}+\lambda \sum_{i=1}^{n}\left\|z_{i}\right\|_{1}, \\
& h_{i}=\exp \left(-\frac{\left\|D z_{i}-x_{i}\right\|_{2}^{2}}{\sigma^{2}}\right) .
\end{aligned}
$$

Here the auxiliary variable, $h_{i}$, will be updated in each update iteration. At each iteration, we update $D$ and $Z$ alternately, which means we firstly fix $D$ and update the sparse code $Z$ with coordinated descent $(\mathrm{CD})$ and then fix $\mathrm{Z}$ to update the dictionary $D$ via stochastic gradient descent (SGD).

As our optimization method is stochastic, we only update the sparse code and dictionary with only one signal for each iteration. In the following paragraphs, we will discuss the optimization in one iteration with only one signal. If a signal, $x=\left(x_{1}, x_{2}, x_{p}\right)^{T} \in R^{p}$, is given, we first update its corresponding sparse code, $z=\left(z_{1}, z_{2}, \ldots, z_{m}\right)$, via CD. Let $z_{l}$ denote the $l$-th entry of $z$ and $d_{k l}$ represents the $k$-th item of $d_{l}$. $d_{l}$ is the $l$-th atom/column of the dictionary $D$. Then, we can calculate the partial derivative of $z_{l}$ in the first part of the function, $f\left(D, z_{i}\right)$, as Eq. 5

$$
\begin{aligned}
\frac{\partial}{\partial z_{l}} c(D, \mathrm{z}) & =\frac{\partial}{\partial z_{l}} \frac{1}{2} h\|D z-x\|_{2}^{2}=-h \sum_{k=1}^{p} d_{k l}\left(x_{k}-\sum_{r=1}^{m} d_{k r} z_{r}\right) \\
& =-h \sum_{k=1}^{p} d_{k l}\left(x_{k}-\sum_{r \neq l}^{m} d_{k r} z_{r}-d_{k l} z_{l}\right) \\
& =-h \sum_{k=1}^{p} d_{k l}\left(x_{k}-\sum_{r \neq l}^{m} d_{k r} z_{r}\right)+h z_{l} \sum_{k=1}^{p}\left(d_{k l}\right)^{2} \\
& =-\rho_{l}+h z_{l} v_{l}
\end{aligned}
$$

where $\rho_{l}=h \sum_{k=1}^{p} d_{k l}\left(x_{k}-\sum_{r \neq l}^{m} d_{k r} z_{r}\right), v_{l}=\sum_{k=1}^{p}\left(d_{k l}\right)^{2}$ and $h$ is the auxiliary variable for the signal. Since we normalize the atom, $d_{l}$, in each iteration, $v_{l}$ can be ignored. Then, we compute the subdifferential of the lasso loss function and equate it to zero to find the optimal solution as follows:

$$
\begin{aligned}
\frac{\partial}{\partial z_{l}} f(D, z) & =\frac{\partial}{\partial z_{l}} c(D, z)+\frac{\partial}{\partial z_{l}} \lambda\|z\|_{1} \\
& =-\rho_{l}+h z_{l} v_{l}+\frac{\partial}{\partial z_{l}} \lambda\|z\|_{1}=0
\end{aligned}
$$

Then, according to the derivative of the $l_{1}$-norm, we can have the following equations.

$$
\begin{cases}-\rho l+h z_{l} \cup_{l}-\lambda=0 & \text { if } Z_{l}<0 \\ -\rho l-\lambda \leq 0 \leq-\rho_{l}+\lambda & \text { if } Z_{l}=0 \\ -\rho l+h z_{l} \cup_{l}+\lambda=0 & \text { if } Z_{l}>0\end{cases}
$$


Finally, we can get the soft thresholding function as:

$$
z_{l}= \begin{cases}\frac{\rho_{l}+\lambda}{h v_{l}} & \text { for } \rho_{l}<-\lambda \\ 0 & \text { for }-\lambda \leq \rho_{l} \leq \lambda \\ \frac{\rho_{l}-\lambda}{h v_{l}} & \text { for } \rho_{l}>\lambda\end{cases}
$$

After we update the sparse code, we propose the following strategy to accelerate the convergence for updating the dictionary $D$. The atom, $d_{l}$ will stay unchanged if $z_{l}$ is zero since $\nabla_{d_{l}}=h(D z-x) z_{l}=0$. Otherwise, as shown in Figure 2, we can update the $l$-th atom of the dictionary $D$ as $d_{l} \leftarrow$ $d_{l}-\gamma_{l} h(D z-x) z_{l} \cdot \gamma_{l}$ is the learning rate provided by an approximation of the Hessian: $R \leftarrow R+z z^{T}$ and $\gamma_{l}$ is given by $1 / r_{l l}$, where $r_{l l}$ is the item at the $l$-th row and $l$-th column of the Hessian matrix $R$. The pseudo-code of the model was shown in Algorithm 1, dubbed as PASCS.

Algorithm 1: Patch analysis-based surface correntropy-induced sparse-coding

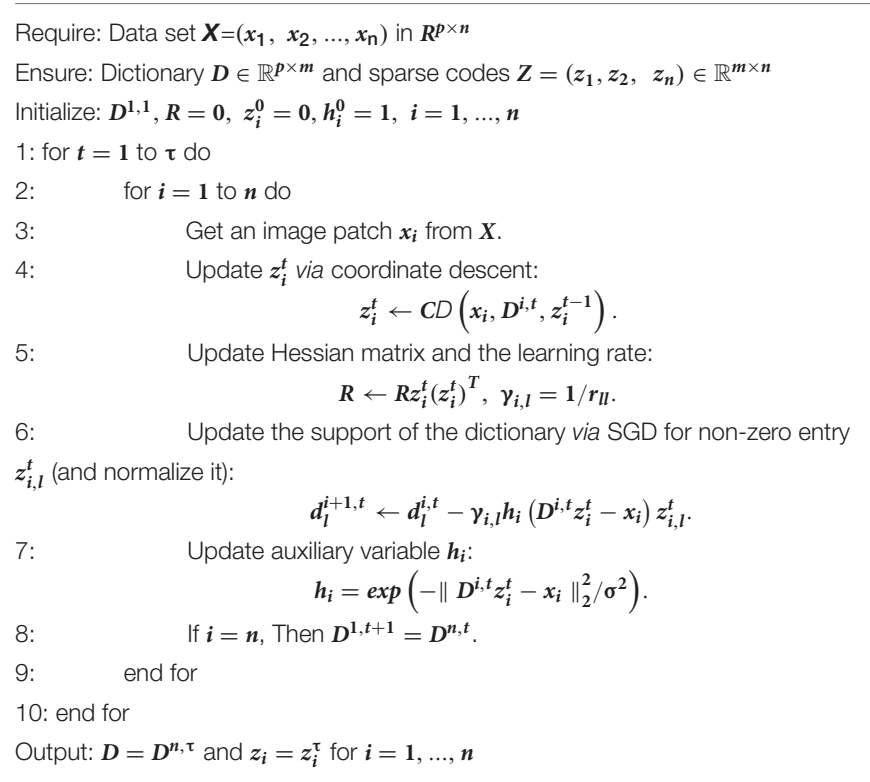

\section{Pooling and Classification}

After we get the sparse code (the dimension is $m$ ) for each patch, the dimensionality of sparse codes for each subject is still too large for classification, which is $\mathrm{m} \times 1,008$. Therefore, we apply Max-pooling to reduce the feature dimensionality for each subject. Max-pooling (Boureau et al., 2010) is a way of taking the most responsive node of a given region of interest and serves as an important layer in the convolutional neural network architecture. In this work, we compute the maximum value of a particular feature over all sparse codes of a subject and generate a new representation for each subject, which is an $m$-dimensional vector. These summary representations are much lower in dimension, compared to using all the extracted surface patch features; this can improve results generalizability via less over-fitting.
With these dimension-reduced features, we choose the random forest algorithm (Liaw and Wiener, 2002) for the binary classification. Random forests are a combination of tree predictors such that each tree depends on the values of a random vector sampled independently and with the same distribution for all trees in the forest. This algorithm adopts a learning process called feature bagging. In this process, we select a random subset of the features several times and then train a decision tree for each subset. If some features are strong predictors of the response, they will be selected in many decision trees and this makes them correlated. In comparison with decision trees, random forests have the same bias but lower variance, which means they can overcome the drawback of overfitting caused by a small data set. For our sparse surface features, when the size of the training set becomes small, diversification becomes more subtle, and the method can better detect these subtle differences. In this project, we use the random forest classifier in the scikit-learn package ${ }^{3}$ with the default settings. Besides, under the imbalanced-data condition (such as $116 \mathrm{~A} \beta+\mathrm{CU}$ and $232 \mathrm{~A} \beta-\mathrm{CU}$ in the ADNI data set), the classifier tends to classify all the training data into the major class, as it aims to maximize training accuracy. Therefore, we adopt random undersampling (Dubey et al., 2014) to balance the numbers of training subjects in the two classes. All the experiments in this work use the same setups for the random forest classifier and random undersampling.

\section{Performance Evaluation Protocol}

Before using hippocampal MMS features for A $\beta$ status classification, we need to apply PASCS-MP to extract sparse codes from these high dimensional MMS features. The performance of PASCS-MP has a close relationship to four key parameters: the patch size, the dimensionality of the learned sparse coding, the regularization parameter for the $l_{1}$-norm $(\lambda)$, and the kernel size $(\sigma)$ in the exponential function (see Eq. 2). Patch-based analysis has been widely used for image segmentation and classification (Kao et al., 2020). Leveraging patches in our MMS can preserve well the properties of the regional morphometry of the hippocampal surface since the vertices that carry strong classification power are always clustered on the surface and a set of such vertices typically has a stronger classification ability compared to using just a single vertex. However, the size of the set of such vertices is unknown. Therefore, we select the vertices by randomly selecting the same number of square patches with different sizes and compared the performance of the final classification accuracy for the different patch sizes. The dimensionality of the learned sparse coding $(m)$ is also the dimensionality of the representation for each subject. The model might miss some significant information if the dimensionality is too low. Also, the representations will contain too much redundant information when the dimensionality is too large. The regularization parameter for the $l_{1}$-norm $(\lambda)$ will control the sparsity of the learned sparse codes. A suitable regularization parameter will select significant features meanwhile reducing noise. The kernel size in the exponential function controls all properties of correntropy. Correntropy is directly related to the probability of how similar two random variables are in

${ }^{3}$ https://scikit-learn.org/ 


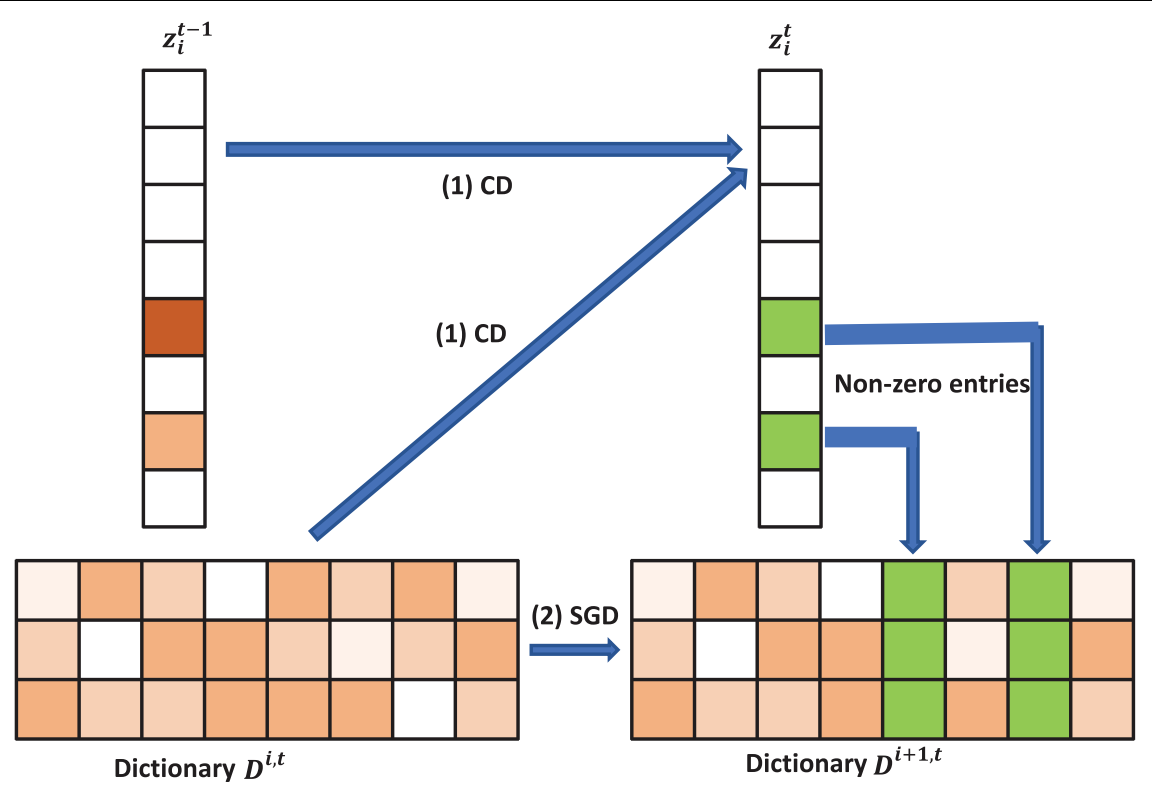

FIGURE 2 | Illustration of one iteration of the proposed Patch Analysis-based Surface Correntropy-induced Sparse-coding (PASCS) algorithm. The input is many $10 \times 10$ patches on each surface based on our multivariate morphometry statistics (MMS). With an image patch $x_{i}$, PASCS performs one step of coordinate descent (CD) to find the support and the sparse code. Meanwhile, PASCS performs a few steps of CD on supports (non-zero entries) to obtain a new sparse code $z_{i}^{k}$. Then, PASCS updates the supports (green boxes in the figure) of the dictionary by stochastic gradient descent (SGD) to obtain a new dictionary $D^{i 1, t}$. Here, $t$ represents the $t$-th epoch; $i$ represents the $i$-th patch.

a neighborhood of the joint space controlled by the kernel bandwidth, i.e., the kernel bandwidth acts as a zoom lens, controlling the observation window over which similarity is assessed. This adjustable window provides an effective mechanism to eliminate the detrimental effect of outliers (Liu et al., 2007).

Thus, we adopt 10 -fold cross-validation to evaluate the classification accuracy on another dataset from ADNI 2 with a series of key parameter candidates and select the optimal parameter setups. The detailed information about the dataset and the key parameter candidates will be introduced in next section. For the 10-fold cross-validation, we randomly shuffle and split the dataset into ten groups. We take one group as the test data set and use the remaining groups to train a model. Then, the candidate model is evaluated using the test data. In this way, we can get a predicted class label for all the samples. Then, the output of each classification experiment is compared to the ground truth, and the accuracy is computed to indicate how many class labels are correctly identified. The key parameters with the highest classification accuracies are selected.

Once we get an optimized PASCS-MP model, we can compare the performances of MMS, volume, and surface area measurements for classifying individuals of different $\mathrm{A} \beta$ status. We use the volume from the left and right hippocampi (i.e., hippocampi in each brain hemisphere) as two features to train the classifier instead of adding them together. The same classification strategy is applied to surface areas from both sides. Moreover, we will compare the classification performances based on PASCS-MP, PASS-MP (Zhang et al., 2016b, 2017b), and SPHARM (Chung et al., 2007, 2008; Shi et al., 2013a).
We evaluate these classification performances with the same 10-fold cross-validation method. Four performance measures: the Accuracy (ACC), Balanced Accuracy (B-ACC), Specificity (SPE), and Sensitivity (SEN) are computed (Hinrichs et al., 2011; Ritter et al., 2015; Zhang et al., 2017b; Bhagwat et al., 2018; Salvatore et al., 2018). We also compute the area-under-thecurve (AUC) of the receiver operating characteristic (ROC) (Fan et al., 2008; La Joie et al., 2013; Bhagwat et al., 2018; Nakamura et al., 2018). By considering these performance measures, we expect the proposed system integrating MMS, PASCS-MP and the binary random forest classifier to perform better than similar classification strategies for identifying individuals with different $\mathrm{A} \beta$ status.

\section{RESULTS}

\section{Key Parameter Estimations for the PASCS-MP Method}

To apply PASCS-MP method on hippocampal MMS, four parameters need to be empirically assigned, namely: the patch size, the dimensionality of the learned sparse coding, the regularization parameter for the $l_{1}$-norm $(\lambda)$ and the kernel size $(\sigma)$ in the exponential function. Selecting suitable parameters will lead to superior performance in refining lower dimensional MMS representations related to $\mathrm{AD}$ pathology. With 10-fold crossvalidation, these key parameters are evaluated from PASCS-MP based classification performance on $109 \mathrm{AD}$ patients and $180 \mathrm{CU}$ subjects of ADNI-2 cohort. To avoid data leakage, these subjects are not used in the following study of $\mathrm{A} \beta$ burden classification. 


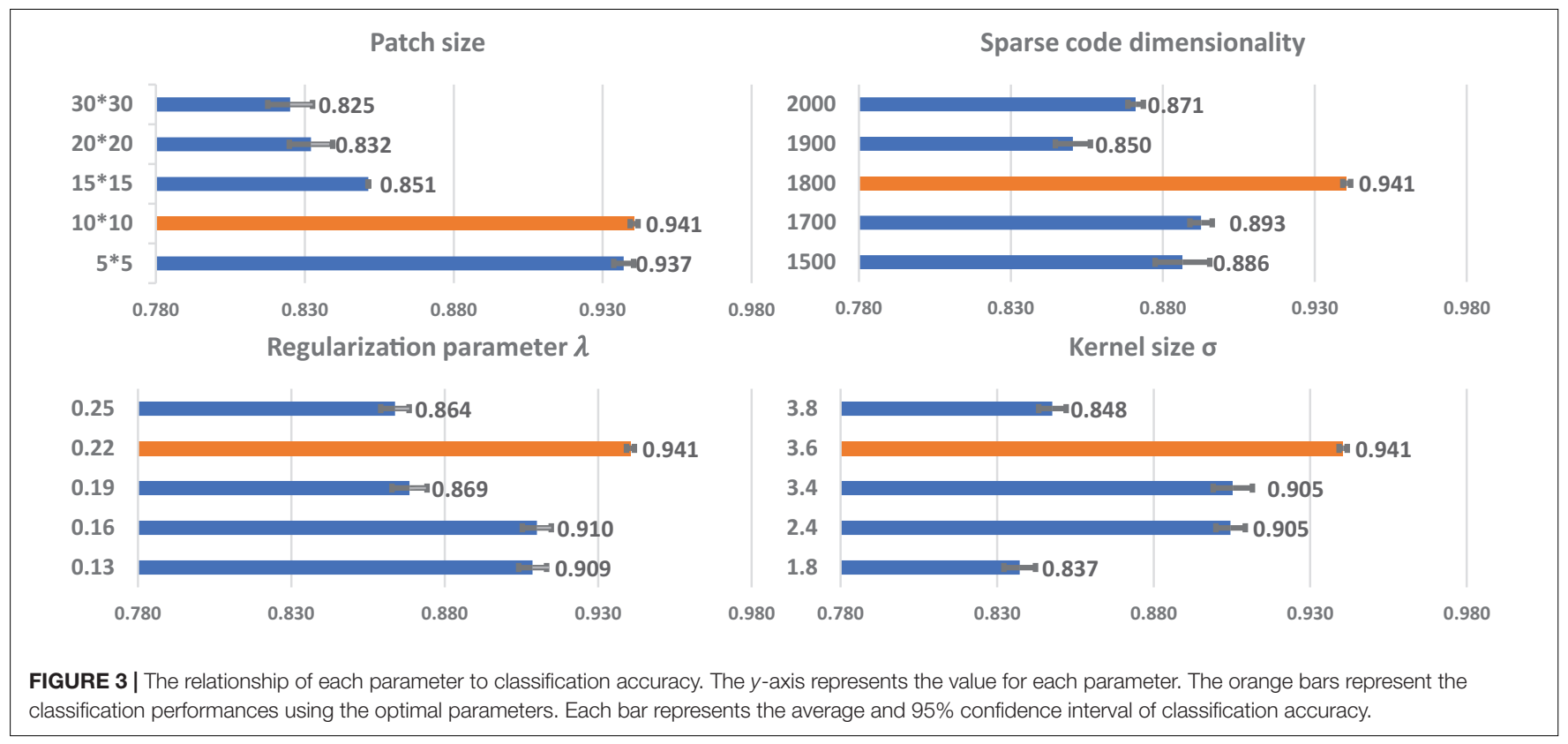

We perform grid search on the data set to explore the optimal parameter settings. In Figure 3, we only illustrate part of the classification accuracy for different values of each parameter in grid search since the combinations of four different parameters will lead to $5^{4}$ results. For each parameter setting, we also repeat 10 -fold cross-validation five times, and the average and 95\% confidence interval of the accuracy are shown in Figure 3. When we evaluate one parameter, we fix the rest parameters. For example, in the first bar chart in Figure 3, we try different patch sizes including $5 \times 5,10 \times 10,15 \times 15,20 \times 20$. and $30 \times 30$ while we fix the sparse code dimensionality as to 1,800 , and set $\lambda$ to 0.22 , and $\sigma$ to 3.6. By testing varied sets of parameters, we find that the optimal patch size is $10 \times 10$, the optimal sparse code dimensionality is 1,800 , the optimal $\lambda$ is 0.22 and the optimal $\sigma$ is 3.6 and these optimal parameters will be adopted in the study of $\mathrm{A} \beta$ burden classification.

\section{Classification of $\mathrm{A} \beta$ Burden}

To explore whether there is a significant gain in classification power with our new system, based on our surface MMS, we generate two different kinds of sparse codes with our previous framework (PASS-MP) (Zhang et al., 2016b, 2017a; Fu et al., 2021) and the new framework (PASCS-MP). The parameter settings for the two sparse coding methods are the same. Additionally, we apply the popular SPHARM method (Chung et al., 2008; Shi et al., 2013a) to calculate hippocampal shape features. Based on these three kinds of feature sets, we apply the random forest classifier to detect individuals with different $A \beta$ status. Moreover, we also examine the classification performances using hippocampal MMS, surface area and volume measures. These classification performances are evaluated using ACC, B-ACC, SPE, and SEN. For each binary classification of ADNI cohort, we repeat the 10 -fold cross-validation five times; the mean and $95 \%$ confident interval of the evaluation measures are calculated as (Vanwinckelen and Blockeel, 2012) and shown in the middle three columns of Table 2.

TABLE 2 | Classification results for four contrasts.

\begin{tabular}{|c|c|c|c|c|}
\hline & $\begin{array}{c}A \beta+A D \text { vs. } \\
A \beta-C U\end{array}$ & $\begin{array}{c}\mathrm{A} \beta+\mathrm{MCl} \text { vs. } \\
\mathrm{A} \beta-\mathrm{MCl}\end{array}$ & $\begin{array}{c}A \beta+C U \text { vs. } A \beta- \\
C U \text { (ADNI) }\end{array}$ & $\begin{array}{c}A \beta+C U \text { vs. } A \beta- \\
C U \text { (OASIS) }\end{array}$ \\
\hline \multicolumn{5}{|l|}{ Area } \\
\hline $\mathrm{ACC}$ & $0.68 \pm 0.01$ & $0.55 \pm 0.02$ & $0.54 \pm 0.01$ & 0.47 \\
\hline B-ACC & $0.69 \pm 0.02$ & $0.55 \pm 0.02$ & $0.54 \pm 0.02$ & 0.43 \\
\hline SPE & $0.66 \pm 0.02$ & $0.54 \pm 0.02$ & $0.55 \pm 0.02$ & 0.49 \\
\hline SEN & $0.71 \pm 0.03$ & $0.56 \pm 0.03$ & $0.53 \pm 0.04$ & 0.37 \\
\hline \multicolumn{5}{|l|}{ Volume } \\
\hline $\mathrm{ACC}$ & $0.71 \pm 0.01$ & $0.53 \pm 0.02$ & $0.50 \pm 0.03$ & 0.51 \\
\hline B-ACC & $0.72 \pm 0.01$ & $0.53 \pm 0.01$ & $0.50 \pm 0.03$ & 0.52 \\
\hline SPE & $0.68 \pm 0.01$ & $0.52 \pm 0.01$ & $0.51 \pm 0.02$ & 0.54 \\
\hline SEN & $0.75 \pm 0.01$ & $0.54 \pm 0.02$ & $0.49 \pm 0.04$ & 0.50 \\
\hline \multicolumn{5}{|c|}{ SPHARM } \\
\hline ACC & $0.71 \pm 0.02$ & $0.56 \pm 0.02$ & $0.52 \pm 0.02$ & 0.60 \\
\hline B-ACC & $0.71 \pm 0.02$ & $0.56 \pm 0.03$ & $0.51 \pm 0.04$ & 0.60 \\
\hline SPE & $0.74 \pm 0.02$ & $0.61 \pm 0.03$ & $0.56 \pm 0.03$ & 0.61 \\
\hline SEN & $0.68 \pm 0.04$ & $0.51 \pm 0.03$ & $0.46 \pm 0.05$ & 0.60 \\
\hline \multicolumn{5}{|c|}{ PASS-MP } \\
\hline ACC & $0.79 \pm 0.01$ & $0.73 \pm 0.02$ & $0.71 \pm 0.02$ & 0.74 \\
\hline B-ACC & $0.79 \pm 0.01$ & $0.73 \pm 0.02$ & $0.70 \pm 0.03$ & 0.73 \\
\hline SPE & $0.78 \pm 0.02$ & $0.75 \pm 0.02$ & $0.73 \pm 0.03$ & 0.74 \\
\hline SEN & $0.79 \pm 0.01$ & $0.72 \pm 0.03$ & $0.67 \pm 0.03$ & 0.73 \\
\hline \multicolumn{5}{|c|}{ PASCS-MP } \\
\hline ACC & $0.91 \pm 0.01$ & $0.89 \pm 0.01$ & $0.79 \pm 0.02$ & 0.81 \\
\hline B-ACC & $0.91 \pm 0.01$ & $0.89 \pm 0.01$ & $0.79 \pm 0.03$ & 0.80 \\
\hline SPE & $0.91 \pm 0.01$ & $0.91 \pm 0.01$ & $0.80 \pm 0.02$ & 0.82 \\
\hline SEN & $0.90 \pm 0.01$ & $0.88 \pm 0.01$ & $0.79 \pm 0.05$ & 0.79 \\
\hline
\end{tabular}

Values are mean $\pm 95 \%$ confident interval where applicable. 
We leverage the OASIS dataset as an external validation set to further evaluate the performance of our new framework. We firstly generate new representations with our proposed PASCSMP for all the CU subjects from ADNI and OASIS cohorts. Then, we train a binary random forest model on the ADNI dataset and test it with the OASIS dataset. Since there is no cross-validation here, there is no confident interval in the last column of Table 2. We also compute the AUC of the ROC. The ROC curve and AUC for these classification tasks are illustrated in Figure 4. This comparison analysis classification performance shows that the combination of PASCS-MP and hippocampal MMS measures have superior performance when detecting individuals with different $A \beta$ status, compared to other similar methods.

\section{DISCUSSION}

In this article, we propose a novel surface feature dimension reduction scheme, PASCS-MP, to correlate the hippocampus MMS with different levels of $A \beta$ burden in individual subjects. We develop a hippocampal structure-based $A \beta$ burden prediction system that involves hippocampal MMS computing, sparse coding and classification modules. We apply the proposed system on two independent datasets, ADNI and OASIS. We have two main findings. Firstly, the hippocampal surface-based MMS measure practically encodes a great deal of neighboring intrinsic geometry information that would otherwise be inaccessible or overlooked in classical hippocampal volume and surface area measures. Experimental results show that the MMS measure provides better classification accuracy than hippocampal volume, surface area measures and SPHARM for detecting the relationships between hippocampal deformations and $A \beta$ positivity. Secondly, we propose a novel sparse coding method, PASCS-MP. It has all the advantages of our previous proposed PASS-MP (Zhang et al., 2016a,b) and improves the follow-up classification performance compared to PASS-MP.

\section{Comparison Analysis of MRI, PET, and BBB}

Amyloid plaques, together with neurofibrillary tangles, are among the earliest signs of $\mathrm{AD}$, appearing before any cognitive impairment and brain structure changes. Measuring $A \beta$ burden at preclinical $\mathrm{AD}$ stages is believed to facilitate identifying individuals appropriate for a given intervention and improving the probability of therapeutic trial success (Brookmeyer et al., 2007; Jack et al., 2016; Sperling et al., 2011a). Brain A $\beta$ pathology can be measured using PET with amyloid directly in the brain, CSF measures, or, more recently, BBBs, which are showing great promise.

Nevertheless, assessment of A $\beta$ pathology using CSF or PET scans can easily become inefficient due to the degree of their acceptance, invasiveness, costs, and/or PET facility availability (Tosun et al., 2016, 2014; Ansart et al., 2020; Pekkala et al., 2020). Developing computational models for detecting $A \beta$ pathology based on less invasive, less costly, and more readily available procedures could help identify a target population with a high prevalence of $A \beta$ pathology. With or without more affordable
$\mathrm{BBBs}$ as an alternative for inferring $\mathrm{A} \beta$ burden in the brain (Bateman et al., 2019; Janelidze et al., 2020; Palmqvist et al., 2020), especially after their diagnosis viabilities are further reduced, sMRI scans will always be an option that is largely accessible, cost-effective, and widely used as a standard-of-care procedure. Structural MRI will also identify pathologies that AD BBB's do not assess, e.g., stroke, tumor, and subdural hematoma. Therefore, a patient will get an MRI scan whether or not they also get BBB. Furthermore, our proposed framework will not only work on AD, but also other diseases like autism spectrum disorder (Fu et al., 2021), major depressive disorder (Yao et al., 2020). Overall, our work will provide important research tools and good insights for computational neuropathology research.

\section{Comparison Analysis of Hippocampal MMS, Volume, and Surface Area}

The hippocampus is a primary target region for studying early $\mathrm{AD}$ progression. Its structure can be measured using the widely used overall hippocampal volume, surface area, and our proposed hippocampal MMS. Our prior studies (Shi et al., 2011; Wang et al., 2011; Li et al., 2016; Dong et al., 2019) show that hippocampal MMS performs robustly in distinguishing clinical groups at different $\mathrm{AD}$ risk levels. In particular, we previously found that hippocampal MMS can detect APOE4 gene dose effects on the hippocampus during the preclinical stage, while the hippocampal volume measure cannot (Dong et al., 2019). A study by Wu et al. (2018) demonstrates that hippocampal MMS performs better than traditional hippocampal volume measures in classifying $151 \mathrm{~A} \beta+\mathrm{AD}$ and $271 \mathrm{~A} \beta-\mathrm{CU}$ subjects.

This work evaluates the performance of the above three hippocampal measurements for predicting $A \beta$ status at the individual subject level. Classification results (see Table 2 and Figure 4) show that hippocampal MMS has better performance as measured by ACC, SPE, SEN, and AUC. These results validate our hypothesis that hippocampal MMS-based analysis methods provide improved statistical accuracy than hippocampal volume and surface area measures in predicting the subjects with different $\mathrm{A} \beta$ status in the $\mathrm{AD}$ continuum. Our prior work (Wang et al., 2011) shows that MMS may offer a surrogate biomarker for PET/CSF A $\beta$ biomarkers. This work further shows it can be used to classify brain $\mathrm{A} \beta$ burden on an individual basis.

\section{Comparative Analysis of PASCS-MP, PASS-MP, and SPHARM}

The MMS measure for brain structures performs well in clinical group comparisons (Wang et al., 2013; Shi et al., 2014, 2015; Li et al., 2016; Yao et al., 2018; Dong et al., 2019, 2020b), and as we have shown, it has the potential to further be applied for individual $A \beta$ classification. To achieve this goal, we need to solve the challenge that the MMS dimension is usually much larger than the number of subjects, i.e., the socalled high dimension, small sample size problem. A reasonable solution is to reduce the feature dimension. Existing feature dimension reduction approaches include feature selection (Jain and Zongker, 1997; Fan et al., 2005), feature extraction (Mika et al., 1999; Jolliffe, 2002; Guyon et al., 2008) and sparse learning 


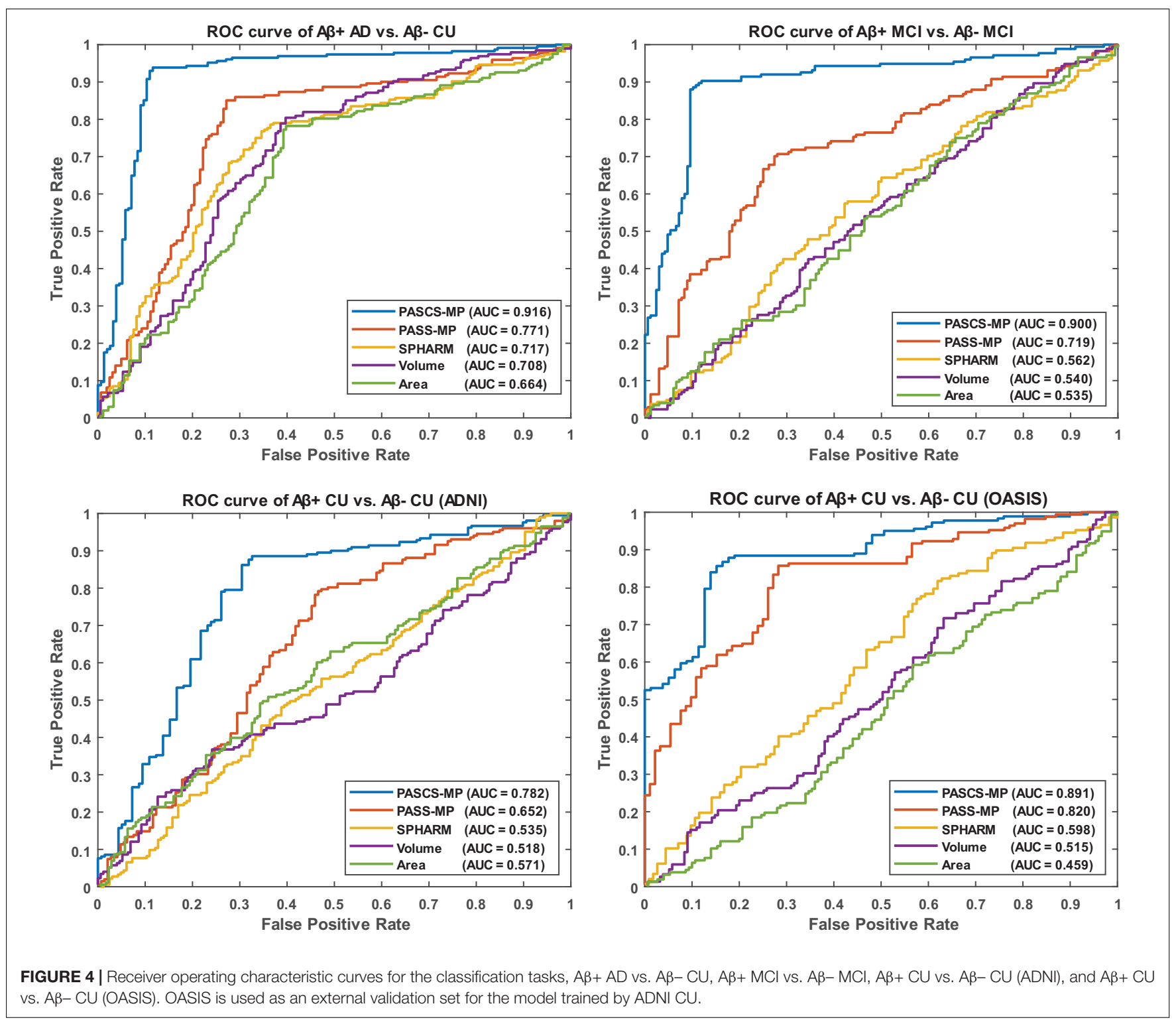

methods (Donoho, 2006; Vounou et al., 2010; Wang et al., 2013). In most cases, information is lost when mapping data into a lower-dimensional space. By defining a better lower-dimensional subspace, this information loss can be limited. Sparse coding (Lee et al., 2007; Mairal et al., 2009) has been previously proposed to learn an over-complete set of basis vectors (also called a dictionary) to represent input vectors efficiently and concisely (Donoho and Elad, 2003). Sparse coding has been shown to be effective for many tasks such as image imprinting (Moody et al., 2012), image deblurring (Yin et al., 2008), super-resolution (Yang et al., 2008), classification (Mairal et al., 2009), functional brain connectivity (Lv et al., 2015, 2017), and structural morphometry analysis (Zhang et al., 2017a).

Our previous studies (Zhang et al., 2016a,b, 2017a) propose a PASS-MP method, consisting of sparse coding (Lee et al., 2007; Mairal et al., 2009), and Max-pooling (LeCun et al., 2015), for surface feature dimension reduction. PASS-MP has excellent impressive performance for the sparse coding of our MMS features. Our prior studies successfully apply these sparse codes in detecting individual brain structure abnormalities and obtain state-of-art performance (Wu et al., 2018; Dong et al., 2020a; Fu et al., 2021).

Even so, there typically exists non-Gaussian and localized sources of noise in surface-based morphometry features, this can dramatically influence the learned dictionary and further lead to poor sparse coding based on the loss function of PASS-MP. The correntropy measure is a very robust method for correcting such sources of noise (Nikolova and Ng, 2006; Liu et al., 2007; He et al., 2012). In this article, we improve upon the PASSMP method by introducing correntropy measures into the loss function (Gui et al., 2017). Therefore, our proposed sparse coding method, PASCS-MP, incorporates all the advantages of PASS-MP and meanwhile improves the classification performance. We also test SPHARM-based hippocampal shape features as they have 
frequently been studied in prior $\mathrm{AD}$ research (e.g., Gerardin et al., 2009; Cuingnet et al., 2011; Gutman et al., 2013). In such an approach, we use a series of SPHARM to model the shapes of the hippocampus segmented by FSL. The SPHARM coefficients are computed using SPHARM-PDM (Spherical Harmonics-Point Distribution Model) software developed by the University of North Carolina and the National Alliance for Medical Imaging Computing (Styner et al., 2006). The classification features are based on these SPHARM coefficients, which are represented by two sets of three-dimensional SPHARM coefficients for each subject (in fact, one set for the hippocampus in each brain hemisphere). In Gerardin et al. (2009), they use a feature selection step because the subject groups are much smaller (fewer than 30 subjects in each group). When the number of subjects is small, the classifier can be more sensitive to uninformative features. In the current study, the number of subjects is relatively large, so a feature selection step is less necessary and may increase the risk of overfitting. We adopt the same approach in Cuingnet et al. (2011), who chose to avoid this selection step. The classification results (see Table 2 and Figure 4) based on PASCS-MP, PASS-MP, and SPHARM meet our expectation that the classification performances based on PASCS-MP have an apparent improvement measured by ACC, B-ACC, SPE, SEN, and AUC.

\section{A $\beta$ Burden Prediction Using MRI Biomarkers}

Beta-amyloid accumulation is a major feature of $\mathrm{AD}$ neuropathology (Brier et al., 2016; Cummings, 2019). Detecting it early and accurately provides a potential opportunity for effective therapeutic interventions before the advanced stages of AD (Tosun et al., 2014). Compared to PET and CSF A $\beta$ measurement techniques, MRI is less expensive (than PET) and less invasive (than both PET and lumbar puncture). AD-related biomarker studies (Sperling et al., 2011b;
Jack and Holtzman, 2013; Jack et al., 2018) have shown that abnormal brain $\mathrm{A} \beta$ accumulation typically precedes detectable structural brain abnormalities. There is emerging literature using MRI biomarkers to predict brain $\mathrm{A} \beta$ burden, and hippocampal structural measurement is one of the major predictors (Tosun et al., 2014, 2016; Ansart et al., 2020; Pekkala et al., 2020). Tosun et al. (2014) applied LASSO penalized logistic regression classifier to MRI-based voxel-wise anatomical shape variation measures and cerebral blood flow measures to predict $A \beta$ positivity in 67 people with early MCI (34 A $\beta+)$; the classification accuracy was 83\%. Ansart et al. (2020) applied LASSO feature selection and a random forest classifier to MRI-based cortical thickness and hippocampal volume measures to classify 596 people with MCI scanned as part of ADNI MCI $(375 \mathrm{~A} \beta+)$; the AUC was 0.80 . Trzepacz et al. (2016) also leveraged logistic regression models with elastic net and hippocampal volume to predict $A \beta$ status in 252 EMCI patients $(120 \mathrm{~A} \beta+)$ and $136 \mathrm{LMCI}$ patients (92 $\mathrm{A} \beta+)$, the AUCs were 0.70 and 0.71 . Our proposed classification framework has a higher ACC $=89 \%$ or AUC $=0.90$ than each of these two studies (Tosun et al., 2014; Ansart et al., 2020) for predicting $A \beta$ status in people with MCI. Of the studies predicting A $\beta$ positivity in CUs, Ansart et al. (2020) applied LASSO feature selection and random forest classifier to MRIderived cortical thickness and hippocampal volume measures to classify 431 ADNI CUs (162 A $\beta+$ ) and 318 INSIGHT CUs $(88 \mathrm{~A} \beta+)$; the AUCs were 0.59 and 0.62 , respectively. Pekkala et al. (2020) used the DSI machine learning algorithm and MRI-based biomarkers (total cortical and gray matter volumes, hippocampus, accumbens, thalamus, and putamen volumes) to predict $\mathrm{A} \beta$ burden in $48 \mathrm{CUs}(20 \mathrm{~A} \beta+)$; the AUC was 0.78 . Tosun et al. (2021) utilized MRI-score extracted from MRI by a trained deep learning model to classify the groups with different $\mathrm{A} \beta$ status in $269 \mathrm{CU}$ patients $(100 \mathrm{~A} \beta+)$. The AUC and ACC were 0.74 and 0.67 . Our proposed classification framework has $\mathrm{AUC}=0.78$ on $348 \mathrm{ADNI} C U \mathrm{~s}(116 \mathrm{~A} \beta+)$ and $\mathrm{AUC}=0.89$ on 260 OASIS CUs $(52 \mathrm{~A} \beta+$ ). Table 3 and Figure 4 present the

TABLE 3 | Studies to impute A $\beta$ status from MRI biomarkers in key clinical groups in AD research.

\begin{tabular}{|c|c|c|c|c|}
\hline Method & Subjects $(A \beta+/-)$ & MRI biomarkers & ACC & AUC \\
\hline \multirow[t]{3}{*}{$\begin{array}{l}\text { PASCS-MP-Random forest classifier (this } \\
\text { work) }\end{array}$} & 342 ADNI MCl (171/171) & $\begin{array}{l}\text { Hippocampal multivariate morphometry statistics } \\
\text { (MMS) }\end{array}$ & $0.89 \pm 0.01$ & 0.90 \\
\hline & 348 ADNI CU (116/232) & & $0.79 \pm 0.02$ & 0.78 \\
\hline & 260 OASIS CU (52/208) & & 0.81 & 0.89 \\
\hline $\begin{array}{l}\text { LASSO penalized logistic regression } \\
\text { classifier (Tosun et al., 2014) }\end{array}$ & 67 early $\mathrm{MCl}(34 / 33)$ & $\begin{array}{l}\text { Voxel-wise anatomical shape variation measures } \\
\text { and cerebral blood flow (including frontoparietal } \\
\text { cortical, hippocampal regions, among others) }\end{array}$ & $0.83 \pm 0.03$ & \\
\hline \multirow{3}{*}{$\begin{array}{l}\text { LASSO feature selection and random forest } \\
\text { classifier (Ansart et al., 2020) }\end{array}$} & 596 ADNI MCI (375/221) & Cortical thickness and hippocampal volume & & 0.80 \\
\hline & 431 ADNI CU (162/269) & & & 0.59 \\
\hline & 318 INSIGHT CU (88/230) & & & 0.62 \\
\hline $\begin{array}{l}\text { Disease State Index machine learning } \\
\text { algorithm (Pekkala et al., 2020) }\end{array}$ & $48 \mathrm{CU}(20 / 28)$ & $\begin{array}{l}\text { Total cortical and gray matter volumes, } \\
\text { hippocampus, accumbens, thalamus, and putamen } \\
\text { volumes }\end{array}$ & & 0.78 \\
\hline \multirow{2}{*}{$\begin{array}{l}\text { Logistic regression analyses including } \\
\text { elastic net classifier (Trzepacz et al., 2016) }\end{array}$} & ADNI EMCI (120/132) & Hippocampal volume & & 0.70 \\
\hline & ADNI LMCI (92/44) & & & 0.71 \\
\hline Random forest (Tosun et al., 2021) & ADNI CU (109/224) & MRI-score extracted by a deep learning model & $0.67 \pm 0.04$ & 0.74 \\
\hline
\end{tabular}


AUC or ACC values from this work and from similar studies predicting $A \beta$ positivity using brain MRI biomarkers. Compared to these similar studies, our proposed classification system only uses hippocampal structural features but still consistently outperforms other recently published methods for predicting $A \beta$ positivity in people with MCI and CUs.

\section{Parameter Selection and Accuracy}

Sparse coding is a representation learning method to generate a sparse representation of the input data in the form of the linear combination of sparse code and a dictionary. Grid search is an effective way to select rational regularization parameters and sparse code dimensionality. However, the accuracy of classification with different parameter settings may not always follow a perfect Gaussian distribution. In Figure 3, the regularization parameter $(0.19)$ and the sparse code dimensionality (1900) have an erratic effect on the classification accuracy. In some other similar work for sparse coding (Xu et al., 2014; Plenge et al., 2015; Quan et al., 2016), the regularization parameter and the sparse code dimensionality also had similar erratic effects on the classification accuracy. It may be due to data insufficiency. Even so, the two cohorts studied in this work arguably had one of the largest imaging data in exiting amyloid prediction research. Further research is warranted to carefully study the relationship between parameter settings and the accuracy results.

\section{Limitations and Future Work}

Despite the promising results are obtained by applying our proposed $A \beta$ positivity classification framework, there are two important caveats. First, when applying the PASCS-MP method to refine MMS, we generally cannot visualize the selected features. To some extent, this decreases the interpretability of the effects, although it is still possible to visualize statistically significant regions as in our prior group difference studies (Shi et al., 2013a; Wang et al., 2013). However, in our recent work (Wu et al., 2020), instead of randomly selecting patches to build the initial dictionary, we use group lasso screening to select the most significant features first. Therefore, the features used in sparse coding may be visualized on the surface map. In the future, we will incorporate this idea into the PASCS-MP framework to make it more interpretable. Second, this work only applies hippocampal MMS to predict $A \beta$ positivity. In future work, we plan to introduce more $\mathrm{AD}$ risk factors (such as demographic information, genetic information, and clinical assessments) (Tosun et al., 2014; Ansart et al., 2020; Pekkala et al., 2020), and more AD regions of interest (ROIs; e.g., ventricles, entorhinal cortex, and temporal lobes) (Brier et al., 2016; Foley et al., 2017; Dong et al., 2020b) into our proposed framework; these additional features are expected to improve the $\mathrm{A} \beta$ positivity prediction.

\section{CONCLUSION}

In this article, we explore the association between hippocampal structures and $\mathrm{A} \beta$ positivity on two independent databases using our hippocampal MMS, PASCS-MP method and a random forest classifier. Compared to traditional hippocampal shape measures, MMS have superior performance for predicting A $\beta$ positivity in the AD continuum. Besides, the proposed PASCSMP outperforms our previous sparse coding method (PASS-MP) on refining MMS features. Compared to similar studies, this work achieves state-of-the-art performance when predicting $A \beta$ positivity based on MRI biomarkers. In the future, we plan to apply this proposed framework to other AD ROIs and further improve the comprehensibility of the framework by visualizing morphometry features selected in the classification.

\section{DATA AVAILABILITY STATEMENT}

The original contributions presented in the study are included in the article/Supplementary Material, further inquiries can be directed to the corresponding author/s.

\section{ETHICS STATEMENT}

The studies involving human participants were reviewed and approved by the Alzheimer's Disease Neuroimaging Initiative. The patients/participants provided their written informed consent to participate in this study.

\section{AUTHOR CONTRIBUTIONS}

JW: methodology, investigation, formal analysis, and writingoriginal draft. QD: conceptualization, investigation, formal analysis, and writing-original draft. JG, JZ, YS, PT, and JY: methodology. KC: methodology and review and editing. RC and ER: review and editing. YW: conceptualization, investigation, supervision, funding acquisition, and writing-review and editing. All authors contributed to the article and approved the submitted version.

\section{FUNDING}

Algorithm development and image analysis for this study were partially supported by the National Institute on Aging (RF1AG051710, R21AG065942, R01AG031581, and P30AG19610), the National Library of Medicine, National Cancer Institute, the National Institute for Biomedical Imaging and Bioengineering (R01EB025032), and the Arizona Alzheimer Consortium. Data collection and sharing for this project was funded by the Alzheimer's Disease Neuroimaging Initiative (ADNI) (National Institutes of Health Grant U01 AG024904) and DoD ADNI (Department of Defense award number W81XWH-12-2-0012). ADNI was funded by the National Institute on Aging, the National Institute of Biomedical Imaging and Bioengineering, and through generous contributions from the following: Alzheimer's Association; Alzheimer's Drug Discovery Foundation; BioClinica, Inc.; Biogen Idec Inc.; Bristol-Myers 
Squibb Company; Eisai Inc.; Elan Pharmaceuticals, Inc.; Eli Lilly and Company; F. Hoffmann-La Roche Ltd. and its affiliated company Genentech, Inc.; GE Healthcare; Innogenetics, N.V.; IXICO Ltd.; Janssen Alzheimer Immunotherapy Research \& Development, LLC.; Johnson \& Johnson Pharmaceutical Research \& Development LLC.; Medpace, Inc.; Merck \& Co., Inc.; Meso Scale Diagnostics, LLC.; NeuroRx Research; Novartis Pharmaceuticals Corporation; Pfizer Inc.; Piramal Imaging; Servier; Synarc Inc; and Takeda Pharmaceutical Company. The Canadian Institutes of Health Research was providing funds to support ADNI clinical sites in Canada. Private sector contributions were facilitated by the Foundation for the National Institutes of Health (www.fnih.org). The grantee organization was the Northern California Institute for Research and Education, and the study was coordinated by the Alzheimer's Disease Cooperative Study at the University of California, San Diego. ADNI data were disseminated by the Laboratory for Neuro Imaging at the University of Southern California.

\section{REFERENCES}

Ansart, M., Epelbaum, S., Gagliardi, G., Colliot, O., Dormont, D., Dubois, B., et al. (2020). Reduction of recruitment costs in preclinical AD trials: validation of automatic pre-screening algorithm for brain amyloidosis. Stat. Methods Med. Res. 29, 151-164. doi: 10.1177/0962280218823036

Apostolova, L. G., Morra, J. H., Green, A. E., Hwang, K. S., Avedissian, C., Woo, E., et al. (2010). Automated 3D mapping of baseline and 12-month associations between three verbal memory measures and hippocampal atrophy in $490 \mathrm{ADNI}$ subjects. Neuroimage 51, 488-499. doi: 10.1016/j.neuroimage.2009.12.125

Bateman, R. J., Blennow, K., Doody, R., Hendrix, S., Lovestone, S., Salloway, S., et al. (2019). Plasma biomarkers of AD emerging as essential tools for drug development: an EU/US CTAD task force report. J. Prev. Alzheimer's Dis. 6, 169-173. doi: 10.14283/jpad.2019.21

Bhagwat, N., Viviano, J. D., Voineskos, A. N., and Chakravarty, M. M. (2018). Modeling and prediction of clinical symptom trajectories in Alzheimer's disease using longitudinal data. PLoS Comput. Biol. 14:e1006376. doi: 10.1371/journal. pcbi.1006376

Boureau, Y. L., Ponce, J., and Lecun, Y. (2010). "A theoretical analysis of feature pooling in visual recognition," in Proceedings of the 27th International Conference on Machine Learning, (Haifa).

Brier, M. R., Gordon, B., Friedrichsen, K., McCarthy, J., Stern, A., Christensen, J., et al. (2016). Tau and Ab imaging, CSF measures, and cognition in Alzheimer's disease. Sci. Transl. Med. 8:338ra66. doi: 10.1126/scitranslmed.aaf2362

Bro-Nielsen, M., and Gramkow, C. (1996). "Fast fluid registration of medical images," in Lecture Notes in Computer Science (Including Subseries Lecture Notes in Artificial Intelligence and Lecture Notes in Bioinformatics), eds K. H. Höhne and R. Kikinis (Berlin: Springer Science+Business Media), doi: 10.1007/ bfb0046964

Brookmeyer, R., Johnson, E., Ziegler-Graham, K., and Arrighi, H. M. (2007). Forecasting the global burden of Alzheimer's disease. Alzheimer's Dement. 3, 186-191. doi: 10.1016/j.jalz.2007.04.381

Cacciaglia, R., Molinuevo, J. L., Falcón, C., Brugulat-Serrat, A., Sánchez-Benavides, G., Gramunt, N., et al. (2018). Effects of APOE - $-\varepsilon 4$ allele load on brain morphology in a cohort of middle-aged healthy individuals with enriched genetic risk for Alzheimer's disease. Alzheimer's Dement. 14, 902-912. doi: 10.1016/j.jalz.2018.01.016

Ching, C. R. K., Abaryan, Z., Santhalingam, V., Zhu, A. H., Bright, J. K., Jahanshad, N., et al. (2020). Sex-Dependent age trajectories of subcortical brain structures: analysis of large-scale percentile models and shape morphometry. bioRxiv Preprint doi: 10.1101/2020.09.30.321711

Chung, M. K., Dalton, K. M., and Davidson, R. J. (2008). Tensor-based cortical surface morphometry via weighted spherical harmonic representation. IEEE Trans. Med. Imaging. 27, 1143-1151. doi: 10.1109/TMI.2008.918338

\section{ACKNOWLEDGMENTS}

Data used in preparation of this article were obtained from the Alzheimer's Disease Neuroimaging Initiative (ADNI) database (adni.loni.usc.edu) and Open Access Series of Imaging Studies (OASIS). As such, the investigators within the ADNI or OASIS contributed to the design and implementation of ADNI or OASIS and/or provided data but did not participate in analysis or writing of this report. A complete listing of ADNI investigators can be found at: http://adni.loni.usc.edu/wp-content/uploads/how_to_ apply/ADNI_Acknowledgement_List.pdf.

\section{SUPPLEMENTARY MATERIAL}

The Supplementary Material for this article can be found online at: https://www.frontiersin.org/articles/10.3389/fnins. 2021.669595/full\#supplementary-material

Chung, M. K., Dalton, K. M., Shen, L., Evans, A. C., and Davidson, R. J. (2007). Weighted Fourier Series Representation and Its Application to Quantifying the Amount of Gray Matter. IEEE Trans. Med. Imaging 26, 566-581. doi: 10.1109/ TMI.2007.892519

Colom, R., Stein, J. L., Rajagopalan, P., Martínez, K., Hermel, D., Wang, Y., et al. (2013). Hippocampal structure and human cognition: key role of spatial processing and evidence supporting the efficiency hypothesis in females. Intelligence 41, 129-140. doi: 10.1016/j.intell.2013.01.002

Costafreda, S. G., Dinov, I. D., Tu, Z., Shi, Y., Liu, C.-Y., Kloszewska, I., et al. (2011). Automated hippocampal shape analysis predicts the onset of dementia in mild cognitive impairment. Neuroimage 56, 212-219.

Crivello, F., Lemaître, H., Dufouil, C., Grassiot, B., Delcroix, N., Tzourio-Mazoyer, N., et al. (2010). Effects of ApoE- 44 allele load and age on the rates of grey matter and hippocampal volumes loss in a longitudinal cohort of 1186 healthy elderly persons. Neuroimage 53, 1064-1069. doi: 10.1016/j.neuroimage.2009. 12.116

Cuingnet, R., Gerardin, E., Tessieras, J., Auzias, G., Lehéricy, S., Habert, M. O., et al. (2011). Automatic classification of patients with Alzheimer's disease from structural MRI: A comparison of ten methods using the ADNI database. Neuroimage 56, 766-781. doi: 10.1016/j.neuroimage.2010.06.013

Cummings, J. (2019). The national institute on aging-Alzheimer's association framework on Alzheimer's disease: application to clinical trials. Alzheimer's Dement. 15, 172-178. doi: 10.1016/j.jalz.2018.05.006

D’Agostino, E., Maes, F., Vandermeulen, D., and Suetens, P. (2003). A viscous fluid model for multimodal non-rigid image registration using mutual information. Med. Image Anal. 7, 565-575. doi: 10.1016/S1361-8415(03)00039-2

Davatzikos, C., Resnick, S. M., Wu, X., Parmpi, P., and Clark, C. M. (2008). Individual patient diagnosis of $\mathrm{AD}$ and FTD via high-dimensional pattern classification of MRI. Neuroimage 41, 1220-1227. doi: 10.1016/j.neuroimage. 2008.03.050

Dong, Q., Zhang, J., Li, Q., Wang, J., Leporé, N., Thompson, P. M., et al. (2020a). Integrating convolutional neural networks and multi-task dictionary learning for cognitive decline prediction with longitudinal images. J. Alzheimers. Dis. 75, 971-992. doi: 10.3233/JAD-190973

Dong, Q., Zhang, W., Stonnington, C. M., Wu, J., Gutman, B. A., Chen, K., et al. (2020b). Applying surface-based morphometry to study ventricular abnormalities of cognitively unimpaired subjects prior to clinically significant memory decline. NeuroImage Clin. 27:102338. doi: 10.1016/j.nicl.2020.102338

Dong, Q., Zhang, W., Wu, J., Li, B., Schron, E. H., McMahon, T., et al. (2019). Applying surface-based hippocampal morphometry to study APOE-E4 allele dose effects in cognitively unimpaired subjects. NeuroImage Clin. 22:101744. doi: 10.1016/j.nicl.2019.101744

Donoho, D. L. (2006). Compressed sensing. IEEE Trans. Inf. Theory 52, 1289-1306. doi: 10.1109/TIT.2006.871582 
Donoho, D. L., and Elad, M. (2003). Optimally sparse representation in general (nonorthogonal) dictionaries via 11 minimization. Proc. Natl. Acad. Sci. U.S.A. 100, 2197-2202.

Dubey, R., Zhou, J., Wang, Y., Thompson, P. M., and Ye, J. (2014). Analysis of sampling techniques for imbalanced data: An $\mathrm{n}=648$ ADNI study. Neuroimage 87, 220-241. doi: 10.1016/j.neuroimage.2013.10.005

Fan, Y., Resnick, S. M., Wu, X., and Davatzikos, C. (2008). Structural and functional biomarkers of prodromal Alzheimer's disease: a high-dimensional pattern classification study. Neuroimage 41, 277-285. doi: 10.1016/j.neuroimage.2008. 02.043

Fan, Y., Shen, D., and Davatzikos, C. (2005). \{C\}lassification of structural images via high-dimensional image warping, robust feature extraction, and $\{\mathrm{S}\}\{\mathrm{V}\}\{\mathrm{M}\}$. Med. Image Comput. Comput. Assist. Interv. 8, 1-8.

Feng, Y., Huang, X., Shi, L., Yang, Y., and Suykens, J. A. K. (2015). Learning with the maximum correntropy criterion induced losses for regression. J. Mach. Learn. Res. 16, 993-1034.

Fleisher, A. S., Chen, K., Liu, X., Roontiva, A., Thiyyagura, P., Ayutyanont, N., et al. (2011). Using positron emission tomography and florbetapir F 18 to image cortical amyloid in patients with mild cognitive impairment or dementia due to Alzheimer disease. Arch. Neurol. 68, 1404-1411. doi: 10.1001/archneurol.2011. 150

Foley, S. F., Tansey, K. E., Caseras, X., Lancaster, T., Bracht, T., Parker, G., et al. (2017). Multimodal brain imaging reveals structural differences in Alzheimer's Disease polygenic risk carriers: a study in healthy young adults. Biol. Psychiatry 81, 154-161. doi: 10.1016/j.biopsych.2016.02.033

Fu, W. J. (1998). Penalized regressions: the bridge versus the lasso? J. Comput. Graph. Stat. 7, 397-416. doi: 10.1080/10618600.1998.10474784

Fu, Y., Zhang, J., Li, Y., Shi, J., Zou, Y., Guo, H., et al. (2021). A novel pipeline leveraging surface-based features of small subcortical structures to classify individuals with autism spectrum disorder. Prog. Neuro-Psychopharmacol. Biol. Psychiatry 104:109989. doi: 10.1016/j.pnpbp.2020.109989

Gerardin, E., Chételat, G., Chupin, M., Cuingnet, R., Desgranges, B., Kim, H.S., et al. (2009). Multidimensional classification of hippocampal shape features discriminates Alzheimer's disease and mild cognitive impairment from normal aging. Neuroimage 47, 1476-1486. doi: 10.1016/j.neuroimage.2009.05.036

Gui, J., Sun, Z., Ji, S., Tao, D., and Tan, T. (2017). Feature selection based on structured sparsity: a comprehensive study. IEEE Trans. Neural Networks Learn. Syst. 28, 1490-1507. doi: 10.1109/TNNLS.2016.2551724

Gutman, B. A., Hua, X., Rajagopalan, P., Chou, Y. Y., Wang, Y., Yanovsky, I., et al. (2013). Maximizing power to track Alzheimer's disease and MCI progression by LDA-based weighting of longitudinal ventricular surface features. Neuroimage 70, 386-401. doi: 10.1016/j.neuroimage.2012.12.052

Guyon, I., Gunn, S., Nikravesh, M., and Zadeh, L. A. (2008). Feature Extraction: Foundations and Applications. Berlin: Springer.

Han, X., Xu, C., and Prince, J. L. (2003). A topology preserving level set method for geometric deformable models. IEEE Trans. Pattern Anal. Mach. Intell. 25, 755-768. doi: 10.1109/TPAMI.2003.1201824

Hardy, J., and Selkoe, D. J. (2002). The amyloid hypothesis of Alzheimer's disease: progress and problems on the road to therapeutics. Science (80-.) 297, 353-356. doi: $10.1126 /$ science. 1072994

He, R., Tan, T., Wang, L., and Zheng, W. S. (2012). "L 2, 1 regularized correntropy for robust feature selection," in Proceedings of the IEEE Computer Society Conference on Computer Vision and Pattern Recognition, (Providence, RI), doi: 10.1109/CVPR.2012.6247966

Hinrichs, C., Singh, V., Xu, G., and Johnson, S. C. (2011). Predictive markers for $\mathrm{AD}$ in a multi-modality framework: An analysis of MCI progression in the ADNI population. Neuroimage 55, 574-589. doi: 10.1016/j.neuroimage.2010. 10.081

Hoppe, H. (1996). "Progressive meshes," in Proceedings of the 23rd Annual Conference on Computer Graphics and Interactive Techniques, SIGGRAPH 1996, (New York, NY: Association for Computing Machinery), doi: 10.1145/237170. 237216

Hyman, B. T. (2011). Amyloid-dependent and amyloid-independent stages of alzheimer disease. Arch. Neurol. 68, 1062-1064. doi: 10.1001/archneurol.2011. 70

Jack, C. R., Bennett, D. A., Blennow, K., Carrillo, M. C., Dunn, B., Haeberlein, S. B., et al. (2018). NIA-AA research framework: toward a biological definition of
Alzheimer's disease. Alzheimer's Dement. 14, 535-562. doi: 10.1016/j.jalz.2018. 02.018

Jack, C. R., Bennett, D. A., Blennow, K., Carrillo, M. C., Feldman, H. H., Frisoni, G. B., et al. (2016). A/T/N: an unbiased descriptive classification scheme for Alzheimer disease biomarkers. Neurology 87, 539-547. doi: 10.1212/WNL. 0000000000002923

Jack, C. R., and Holtzman, D. M. (2013). Biomarker modeling of alzheimer's disease. Neuron 80, 1347-1358. doi: 10.1016/j.neuron.2013.12.003

Jain, A., and Zongker, D. (1997). Feature selection: evaluation, application, and small sample performance. Pattern Anal. Mach. Intell. IEEE Trans. 19, 153-158.

Janelidze, S., Mattsson, N., Palmqvist, S., Smith, R., Beach, T. G., Serrano, G. E., et al. (2020). Plasma P-tau181 in Alzheimer's disease: relationship to other biomarkers, differential diagnosis, neuropathology and longitudinal progression to Alzheimer's dementia. Nat. Med. 26, 379-386. doi: 10.1038/ s41591-020-0755-1

Jolliffe, I. T. (2002). Principal Component Analysis, Second Edn. New York, NY: Springer-Verlag, doi: 10.2307/1270093

Kao, P. Y., Shailja, F., Jiang, J., Zhang, A., Khan, A., Chen, J. W., et al. (2020). Improving patch-based convolutional neural networks for MRI Brain Tumor Segmentation by Leveraging Location information. Front. Neurosci. 3:1449. doi: $10.3389 /$ fnins. 2019.01449

Klunk, W. E., Koeppe, R. A., Price, J. C., Benzinger, T. L., Devous, M. D., Jagust, W. J., et al. (2015). The Centiloid project: Standardizing quantitative amyloid plaque estimation by PET. Alzheimers Dement. 11, 1-15.e1-4. doi: 10.1016/j. jalz.2014.07.003

La Joie, R., Perrotin, A., De La Sayette, V., Egret, S., Doeuvre, L., Belliard, S., et al. (2013). Hippocampal subfield volumetry in mild cognitive impairment, Alzheimer's disease and semantic dementia. NeuroImage Clin. 3, 155-162. doi: 10.1016/j.nicl.2013.08.007

LeCun, Y., Bengio, Y., and Hinton, G. (2015). Deep learning. Nature 521, 436-444. doi: $10.1038 /$ nature 14539

Lee, H., Battle, A., Raina, R., and Ng, A. Y. (2007). "Efficient sparse coding algorithms," in Advances in Neural Information Processing Systems, eds B. Schölkopf, J. Platt, and T. Hofmann (Cambridge, MA: MIT Press), doi: 10.7551/ mitpress/7503.003.0105

Lee, Y. K., Hou, S. W., Lee, C. C., Hsu, C. Y., Huang, Y. S., and Su, Y. C. (2013). Increased risk of dementia in patients with mild traumatic brain injury: a nationwide cohort study. PLoS One 8:e62422. doi: 10.1371/journal.pone. 0062422

Leow, A., Huang, S. C., Geng, A., Becker, J., Davis, S., Toga, A., et al. (2005). "Inverse consistent mapping in 3D deformable image registration: its construction and statistical properties," in Lecture Notes in Computer Science, eds G. E. Christensen and M. Sonka (Berlin: Springer), doi: 10.1007/115057 30_41

Li, B., Shi, J., Gutman, B. A., Baxter, L. C., Thompson, P. M., Caselli, R. J., et al. (2016). Influence of APOE genotype on hippocampal atrophy over timeAn N=1925 surface-based ADNI study. PLoS One 11:e0152901. doi: 10.1371/ journal.pone.0152901

Liaw, A., and Wiener, M. (2002). Classification and regression by RandomForest. $R$ News 2, 18-22.

Lin, B., Li, Q., Sun, Q., Lai, M.-J., Davidson, I., Fan, W., et al. (2014). Stochastic Coordinate Coding and Its Application for Drosophila Gene Expression Pattern Annotation. arXiv Preprint

Liu, W., Pokharel, P. P., and Principe, J. C. (2007). Correntropy: properties and applications in non-Gaussian signal processing. IEEE Trans. Signal Process. 55, 5286-5298. doi: 10.1109/TSP.2007.896065

Loop, C. (1987). Smooth Subdivision Surfaces Based on Triangles. Masters. Thesis, The University of Utah, Salt Lake.

Lorensen, W. E., and Cline, H. E. (1987). "Marching cubes: A high resolution 3D surface construction algorithm," in Proceedings of the 14th Annual Conference on Computer Graphics and Interactive Techniques, SIGGRAPH 1987, (New York, NY: Association for Computing Machinery), doi: 10.1145/37401. 37422

Luders, E., Thompson, P. M., Kurth, F., Hong, J. Y., Phillips, O. R., Wang, Y., et al. (2013). Global and regional alterations of hippocampal anatomy in long-term meditation practitioners. Hum. Brain Mapp. 34, 3369-3375. doi: 10.1002/hbm. 22153 
Lv, J., Jiang, X., Li, X., Zhu, D., Zhang, S., Zhao, S., et al. (2015). $\{$ H $\}$ olistic atlases of functional networks and interactions reveal reciprocal organizational architecture of cortical function. IEEE Trans. Biomed. Eng. 62, 1120-1131.

Lv, J., Lin, B., Li, Q., Zhang, W., Zhao, Y., Jiang, X., et al. (2017). Task fMRI data analysis based on supervised stochastic coordinate coding. Med. Image Anal. 38, 1-16. doi: 10.1016/j.media.2016.12.003

Mairal, J., Bach, F., Ponce, J., and Sapiro, G. (2009). "Online dictionary learning for sparse coding," in Proceedings of the 26th Annual International Conference on Machine Learning, (New Yor, kNY: Association for Computing Machinery), 689-696. doi: 10.1145/1553374.1553463

Marcus, D. S., Fotenos, A. F., Csernansky, J. G., Morris, J. C., and Buckner, R. L. (2010). Open access series of imaging studies: longitudinal MRI Data in Nondemented and demented older adults. J. Cogn. Neurosci. 22, 2677-2684. doi: 10.1162/jocn.2009.21407

Mika, S., Ratsch, G., Weston, J., Scholkopf, B., and Muller, K. R. (1999). "Fisher discriminant analysis with kernels," in Proceedings of the Neural Networks for Signal Processing - Proceedings of the IEEE Workshop, (Madison, WI), doi: 10.1109/nnsp.1999.788121

Monje, M., Thomason, M. E., Rigolo, L., Wang, Y., Waber, D. P., Sallan, S. E., et al. (2013). Functional and structural differences in the hippocampus associated with memory deficits in adult survivors of acute lymphoblastic leukemia. Pediatr. Blood Cancer. 60, 293-300. doi: 10.1002/pbc.24263

Moody, D. I., Brumby, S. P., Rowland, J. C., and Gangodagamage, C. (2012). "Unsupervised land cover classification in multispectral imagery with sparse representations on learned dictionaries," in Proceedings of the Applied Imagery Pattern Recognition Workshop, (Washington, DC), doi: 10.1109/AIPR.2012. 6528190

Morra, J. H., Tu, Z., Apostolova, L. G., Green, A. E., Avedissian, C., Madsen, S. K., et al. (2009). Automated 3D mapping of hippocampal atrophy and its clinical correlates in 400 subjects with Alzheimer's disease, mild cognitive impairment, and elderly controls. Hum. Brain Mapp. 30, 2766-2788. doi: 10.1002/hbm. 20708

Nakamura, A., Kaneko, N., Villemagne, V. L., Kato, T., Doecke, J., Doré, V., et al. (2018). High performance plasma amyloid- $\beta$ biomarkers for Alzheimer's disease. Nature 554, 249-254. doi: 10.1038/nature25456

Navitsky, M., Joshi, A. D., Kennedy, I., Klunk, W. E., Rowe, C. C., Wong, D. F., et al. (2018). Standardization of amyloid quantitation with florbetapir standardized uptake value ratios to the Centiloid scale. Alzheimers Dement. 14, 1565-1571. doi: 10.1016/j.jalz.2018.06.1353

Nikolova, M., and Ng, M. K. (2006). Analysis of half-quadratic minimization methods for signal and image recovery. SIAM J. Sci. Comput. 27, 937-966. doi: $10.1137 / 030600862$

Olshausen, B. A., and Field, D. J. (1997). Sparse coding with an overcomplete basis set: a strategy employed by V1? Vision Res. 37, 3311-3325. doi: 10.1016/S00426989(97)00169-7

Palmqvist, S., Janelidze, S., Quiroz, Y. T., Zetterberg, H., Lopera, F., Stomrud, E., et al. (2020). Discriminative accuracy of plasma Phospho-tau217 for Alzheimer Disease vs other neurodegenerative disorders. JAMA 324:772. doi: 10.1001/ jama.2020.12134

Paquette, N., Shi, J., Wang, Y., Lao, Y., Ceschin, R., Nelson, M. D., et al. (2017). Ventricular shape and relative position abnormalities in preterm neonates. NeuroImage. Clin. 15, 483-493. doi: 10.1016/j.nicl.2017.05.025

Patenaude, B., Smith, S. M., Kennedy, D. N., and Jenkinson, M. (2011). A Bayesian model of shape and appearance for subcortical brain segmentation. Neuroimage 56, 907-922. doi: 10.1016/j.neuroimage.2011.02.046

Pekkala, T., Hall, A., Ngandu, T., Gils, M., van Helisalmi, S., Hänninen, T., et al. (2020). Detecting amyloid positivity in elderly with increased risk of cognitive decline. Front. Aging Neurosci. 12:228. doi: 10.3389/fnagi.2020.00228

Pizer, S. M., Fritsch, D. S., Yushkevich, P. A., Johnson, V. E., and Chaney, E. L. (1999). Segmentation, registration, and measurement of shape variation via image object shape. IEEE Trans. Med. Imaging 18, 851-865. doi: 10.1109/42. 811263

Plenge, E., Klein, S. S., Niessen, W. J., and Meijering, E. (2015). Multiple sparse representations classification. PLoS One 10:e0131968. doi: 10.1371/journal.pone.0131968

Qiu, A., Taylor, W. D., Zhao, Z., MacFall, J. R., Miller, M. I., Key, C. R., et al. (2009). APOE related hippocampal shape alteration in geriatric depression. Neuroimage 44, 620-626.
Quan, Y., Xu, Y., Sun, Y., Huang, Y., and Ji, H. (2016). "Sparse coding for classification via discrimination ensemble," in proceedings of the IEEE Computer Society Conference on Computer Vision and Pattern Recognition. doi: 10.1109/ CVPR.2016.629

Reiter, K., Nielson, K. A., Durgerian, S., Woodard, J. L., Smith, J. C., Seidenberg, M., et al. (2017). Five-year longitudinal brain volume change in healthy elders at genetic risk for Alzheimer's Disease. J. Alzheimers Dis. 55, 1363-1377. doi: 10.3233/JAD- 160504

Rey, D., Subsol, G., Delingette, H., and Ayache, N. (2002). Automatic detection and segmentation of evolving processes in $3 \mathrm{D}$ medical images: application to multiple sclerosis. Med. Image Anal. 6, 163-179. doi: 10.1016/S1361-8415(02) 00056-7

Ritter, K., Schumacher, J., Weygandt, M., Buchert, R., Allefeld, C., and Haynes, J. D. (2015). Multimodal prediction of conversion to Alzheimer's disease based onincomplete biomarkers. Alzheimers Dement. 1, 206-215. doi: 10.1016/ j.dadm.2015.01.006

Rowe, C. C., Doré, V., Jones, G., Baxendale, D., Mulligan, R. S., Bullich, S., et al. (2017). 18F-Florbetaben PET beta-amyloid binding expressed in Centiloids. Eur. J. Nucl. Med. Mol. Imaging 44, 2053-2059. doi: 10.1007/s00259-0173749-6

Salvatore, C., Cerasa, A., and Castiglioni, I. (2018). MRI characterizes the progressive course of $\mathrm{AD}$ and predicts conversion to Alzheimer's Dementia 24 months before probable diagnosis. Front. Aging Neurosci. 10:135. doi: 10.3389/ fnagi.2018.00135

Shen, L., Firpi, H. A., Saykin, A. J., and West, J. D. (2009). Parametric surface modeling and registration for comparison of manual and automated segmentation of the hippocampus. Hippocampus 19, 588-595. doi: 10.1002/ hipo. 20613

Shi, J., Leporé, N., Gutman, B. A., Thompson, P. M., Baxter, L. C., Caselli, R. J., et al. (2014). Genetic influence of apolipoprotein E4 genotype on hippocampal morphometry: An $\mathrm{N}=725$ surface-based Alzheimer's disease neuroimaging initiative study. Hum. Brain Mapp. 35, 3903-3918. doi: 10.1002/hbm.22447

Shi, J., Stonnington, C. M., Thompson, P. M., Chen, K., Gutman, B., Reschke, C., et al. (2015). Studying ventricular abnormalities in mild cognitive impairment with hyperbolic Ricci flow and tensor-based morphometry. Neuroimage 104, 1-20. doi: 10.1016/j.neuroimage.2014.09.062

Shi, J., Thompson, P. M., Gutman, B., and Wang, Y. (2013a). Surface fluid registration of conformal representation: application to detect disease burden and genetic influence on hippocampus. Neuroimage 78, 111-134. doi: 10.1016/ j.neuroimage.2013.04.018

Shi, J., Thompson, P. M., and Wang, Y. (2011). "Human brain mapping with conformal geometry and multivariate tensor-based morphometry," in Lecture Notes in Computer Science (Including Subseries Lecture Notes in Artificial Intelligence and Lecture Notes in Bioinformatics), eds T. Liu, D. Shen, L. Ibanez, and X. Tao (Berlin: Springer), 126-134. doi: 10.1007/978-3-642-24446-9_16

Shi, J., Wang, Y., Ceschin, R., An, X., Lao, Y., Vanderbilt, D., et al. (2013b). A multivariate surface-based analysis of the putamen in premature newborns: regional differences within the ventral striatum. PLoS One. 8:e66736. doi: 10. 1371/journal.pone.0066736

Sperling, R. A., Aisen, P. S., Beckett, L. A., Bennett, D. A., Craft, S., Fagan, A. M., et al. (2011a). Toward defining the preclinical stages of Alzheimer's disease: recommendations from the National Institute on Aging-Alzheimer's Association workgroups on diagnostic guidelines for Alzheimer's disease. Alzheimers Dement. 7, 280-292. doi: 10.1016/j.jalz.2011.03.003

Sperling, R. A., Jack, C. R., Black, S. E., Frosch, M. P., Greenberg, S. M., Hyman, B. T., et al. (2011b). Amyloid-related imaging abnormalities in amyloid-modifying therapeutic trials: recommendations from the Alzheimer's association research roundtable workgroup. Alzheimers Dement. 7, 367-385. doi: 10.1016/j.jalz.2011.05.2351

Styner, M., Lieberman, J. A., Pantazis, D., and Gerig, G. (2004). Boundary and medial shape analysis of the hippocampus in schizophrenia. Med. Image Anal. 8, 197-203. doi: 10.1016/j.media.2004.06.004

Styner, M., Oguz, I., Xu, S., Brechbühler, C., Pantazis, D., Levitt, J. J., et al. (2006). Framework for the statistical shape analysis of brain structures using SPHARM-PDM. Insight J. 1071, 242-250.

Su, Y., Blazey, T. M., Snyder, A. Z., Raichle, M. E., Marcus, D. S., Ances, B. M., et al. (2015). Partial volume correction in quantitative amyloid imaging. Neuroimage 107, 55-64. doi: 10.1016/j.neuroimage.2014.11.058 
Su, Y., Flores, S., Hornbeck, R. C., Speidel, B., Vlassenko, A. G., Gordon, B. A., et al. (2018). Utilizing the Centiloid scale in cross-sectional and longitudinal PiB PET studies. NeuroImage Clin. 19, 406-416. doi: 10.1016/j.nicl.2018.04.022

Su, Y., Flores, S., Wang, G., Hornbeck, R. C., Speidel, B., Joseph-Mathurin, N., et al. (2019). Comparison of Pittsburgh compound B and florbetapir in crosssectional and longitudinal studies. Alzheimers Dement. Diagnosis Assess. Dis. Monit. 11, 180-190. doi: 10.1016/j.dadm.2018.12.008

Sun, D., van Erp, T. G. M., Thompson, P. M., Bearden, C. E., Daley, M., Kushan, L., et al. (2009). Elucidating a magnetic resonance imaging-based neuroanatomic biomarker for psychosis: classification analysis using probabilistic brain atlas and machine learning algorithms. Biol. Psychiatry 66, 1055-1060. doi: 10.1016/ j.biopsych.2009.07.019

Thompson, P. M., Gledd, J. N., Woods, R. P., MacDonald, D., Evans, A. C., and Toga, A. W. (2000). Growth patterns in the developing brain detected by using continuum mechanical tensor maps. Nature 404, 190-193.

Thompson, P. M., Hayashi, K. M., De Zubicaray, G. I., Janke, A. L., Rose, S. E., Semple, J., et al. (2004). Mapping hippocampal and ventricular change in Alzheimer disease. Neuroimage 22, 1754-1766. doi: 10.1016/j.neuroimage.2004. 03.040

Tosun, D., Chen, Y.-F., Yu, P., Sundell, K. L., Suhy, J., Siemers, E., et al. (2016). Amyloid status imputed from a multimodal classifier including structural MRI distinguishes progressors from nonprogressors in a mild Alzheimer's disease clinical trial cohort. Alzheimers Dement. 12, 977-986. doi: 10.1016/j.jalz.2016. 03.009

Tosun, D., Joshi, S., and Weiner, M. W. (2014). Multimodal MRI-based imputation of the A $\beta+$ in early mild cognitive impairment. Ann. Clin. Transl. Neurol. 1, 160-170. doi: 10.1002/acn3.40

Tosun, D., Veitch, D., Aisen, P., Jack, C. R., Jagust, W. J., Petersen, R. C., et al. (2021). . Detection of $\beta$-amyloid positivity in Alzheimer's Disease Neuroimaging Initiative participants with demographics, cognition, MRI and plasma biomarkers. Brain Commun. 3:fcab008. doi: 10.1093/braincomms/ fcab008

Trzepacz, P. T., Hochstetler, H., Yu, P., Castelluccio, P., Witte, M. M., Dellagnello, G., et al. (2016). Relationship of hippocampal volume to amyloid burden across diagnostic stages of Alzheimer's Disease. Dement. Geriatr. Cogn. Disord. 41, 68-79. doi: 10.1159/000441351

Vanwinckelen, G., and Blockeel, H. (2012). "On estimating model accuracy with repeated cross-validation," in Proceedings of the 21st Belgian-Dutch Conference on Machine Learning, (Ghent).

Vounou, M., Nichols, T. E., Montana, G., and Initiative, A. D. N. (2010). Discovering genetic associations with high-dimensional neuroimaging phenotypes: a sparse reduced-rank regression approach. Neuroimage 53, $1147-1159$.

Wang, Y., Chan, T. F., Toga, A. W., and Thompson, P. M. (2009). "Multivariate tensor-based brain anatomical surface morphometry via holomorphic oneforms," in Lecture Notes in Computer Science (Including Subseries Lecture Notes in Artificial Intelligence and Lecture Notes in Bioinformatics), eds G. Z. Yang, D. Hawkes, D. Rueckert, A. Noble, and C. Taylor (Berlin: Springer), doi: 10.1007/ 978-3-642-04268-3_42

Wang, Y., Lui, L. M., Gu, X., Hayashi, K. M., Chan, T. F., Toga, A. W., et al. (2007). Brain surface conformal parameterization using riemann surface structure. IEEE Trans. Med. Imaging. 26, 853-865. doi: 10.1109/TMI.2007.895464

Wang, Y., Shi, J., Yin, X., Gu, X., Chan, T. F., Yau, S. T., et al. (2012). Brain surface conformal parameterization with the ricci flow. IEEE Trans. Med. Imaging. 31, 251-264. doi: 10.1109/TMI.2011.2168233

Wang, Y., Song, Y., Rajagopalan, P., An, T., Liu, K., Chou, Y. Y., et al. (2011). Surface-based TBM boosts power to detect disease effects on the brain: An N=804 ADNI study. Neuroimage 56, 1993-2010. doi: 10.1016/j.neuroimage. 2011.03.040

Wang, Y., Yuan, L., Shi, J., Greve, A., Ye, J., Toga, A. W., et al. (2013). Applying tensor-based morphometry to parametric surfaces can improve MRI-based disease diagnosis. Neuroimage 74, 209-230. doi: 10.1016/j.neuroimage.2013. 02.011

Wang, Y., Zhang, J., Gutman, B., Chan, T. F., Becker, J. T., Aizenstein, H. J., et al. (2010). Multivariate tensor-based morphometry on surfaces: application to mapping ventricular abnormalities in HIV/AIDS. Neuroimage 49, 2141-2157. doi: 10.1016/j.neuroimage.2009.10.086
Wu, J., Zhang, J., Li, Q., Su, Y., Chen, K., Reiman, E. M., et al. (2020). Patch-based surface morphometry feature selection with federated group lasso regression. SPIE-Intl. Soc. Optical Eng. 11583:1158304. doi: 10.1117/12.2575984

Wu, J., Zhang, J., Shi, J., Chen, K., Caselli, R. J., Reiman, E. M., et al. (2018), "Hippocampus morphometry study on pathology-confirmed Alzheimer's disease patients with surface multivariate morphometry statistics," in Proceedings of the IEEE 15th International Symposium on Biomedical Imaging, (Washington, DC), doi: 10.1109/ISBI.2018.8363870

Xu, J., Yang, G., Yin, Y., Man, H., and He, H. (2014). Sparse-representation-based classification with structure-preserving dimension reduction. Cognit. Comput. 6, 608-621. doi: 10.1007/s12559-014-9252-5

Yang, J., Wright, J., Huang, T., and Yi, M. (2008). "Image super-resolution as sparse representation of raw image patches," in Proceedings of the 2008 IEEE Conference on Computer Vision and Pattern Recognition, (Piscataway, NJ: IEEE), 1-8. doi: 10.1109/CVPR.2008.4587647

Yao, Z., Fu, Y., Wu, J., Zhang, W., Yu, Y., Zhang, Z., et al. (2018). Morphological changes in subregions of hippocampus and amygdala in major depressive disorder patients. Brain Imaging Behav. 14, 653-667. doi: 10.1007/s11682-0180003-1

Yao, Z., Fu, Y., Wu, J., Zhang, W., Yu, Y., Zhang, Z., et al. (2020). Morphological changes in subregions of hippocampus and amygdala in major depressive disorder patients. Brain Imaging Behav. 14, 653-667.

Yin, W., Osher, S., Goldfarb, D., and Darbon, J. (2008). Bregman iterative Algorithms for \$lell_1\$-Minimization with applications to compressed sensing. SIAM J. Imaging Sci. 1, 143-168. doi: 10.1137/070703983

Younes, L., Ratnanather, J. T., Brown, T., Aylward, E., Nopoulos, P., Johnson, H., et al. (2014). Regionally selective atrophy of subcortical structures in prodromal HD as revealed by statistical shape analysis. Hum Brain Mapp. 35, 792-809. doi: 10.1002/hbm.22214

Zhang, J., Fan, Y., Li, Q., Thompson, P. M., Ye, J., and Wang, Y. (2017a). "Empowering cortical thickness measures in clinical diagnosis of Alzheimer's disease with spherical sparse coding," in Proceedings of the IEEE 14th International Symposium on Biomedical Imaging, (Melbourne, VIC), doi: 10. 1109/ISBI.2017.7950557

Zhang, J., Li, Q., Caselli, R. J., Thompson, P. M., Ye, J., and Wang, Y. (2017b). Multi-source Multi-target Dictionary Learning for Prediction of Cognitive Decline. Cham: Springer, 184-197. doi: 10.1007/978-3-319-59050-9_15

Zhang, J., Shi, J., Stonnington, C., Li, Q., Gutman, B. A., Chen, K., et al. (2016a). "Hyperbolic space sparse coding with its application on prediction of Alzheimer's disease in mild cognitive impairment," in Lecture Notes in Computer Science (Including Subseries Lecture Notes in Artificial Intelligence and Lecture Notes in Bioinformatics), eds S. Ourselin, L. Joskowicz, M. Sabuncu, G. Unal, and W. Wells (Cham: Springer), doi: 10.1007/978-3-319-467 20-7_38

Zhang, J., Stonnington, C., Li, Q., Shi, J., Bauer, R. J., Gutman, B. A., et al. (2016b). "Applying sparse coding to surface multivariate tensor-based morphometry to predict future cognitive decline," in Proceedings of the IEEE 13th International Symposium on Biomedical Imaging, (Prague), doi: 10.1109/ISBI.2016.749 3350

Conflict of Interest: The authors declare that the research was conducted in the absence of any commercial or financial relationships that could be construed as a potential conflict of interest.

Publisher's Note: All claims expressed in this article are solely those of the authors and do not necessarily represent those of their affiliated organizations, or those of the publisher, the editors and the reviewers. Any product that may be evaluated in this article, or claim that may be made by its manufacturer, is not guaranteed or endorsed by the publisher.

Copyright $\odot 2021 \mathrm{Wu}$, Dong, Gui, Zhang, Su, Chen, Thompson, Caselli, Reiman, Ye and Wang. This is an open-access article distributed under the terms of the Creative Commons Attribution License (CC BY). The use, distribution or reproduction in other forums is permitted, provided the original author(s) and the copyright owner(s) are credited and that the original publication in this journal is cited, in accordance with accepted academic practice. No use, distribution or reproduction is permitted which does not comply with these terms. 